\title{
Analytic tools to bound the criticality at the outer boundary of the period annulus
}

\author{
F. Mañosas ${ }^{1}$, D. Rojas ${ }^{1}$ and J. Villadelprat ${ }^{2}$ \\ ${ }^{1}$ Departament de Matemàtiques, \\ Universitat Autònoma de Barcelona, Barcelona, Spain \\ ${ }^{2}$ Departament d'Enginyeria Informàtica i Matemàtiques, \\ Universitat Rovira i Virgili, Tarragona, Spain
}

\begin{abstract}
In this paper we consider planar potential differential systems and we study the bifurcation of critical periodic orbits from the outer boundary of the period annulus of a center. In the literature the usual approach to tackle this problem is to obtain a uniform asymptotic expansion of the period function near the outer boundary. The novelty in the present paper is that we directly embed the derivative of the period function into a collection of functions that form a Chebyshev system near the outer boundary. We obtain in this way explicit sufficient conditions in order that at most $n \geqslant 0$ critical periodic orbits bifurcate from the outer boundary. These theoretical results are then applied to study the bifurcation diagram of the period function of the family $\ddot{x}=x^{p}-x^{q}, p, q \in \mathbb{R}$ with $p>q$.
\end{abstract}

\section{Introduction and setting of the problem}

This paper is concerned with the period function of centers of planar differential systems. A singular point $p$ of an analytic differential system

$$
\left\{\begin{array}{l}
\dot{x}=f(x, y) \\
\dot{y}=g(x, y)
\end{array}\right.
$$

is a center if it has a punctured neighbourhood that consists entirely of periodic orbits surrounding $p$. The largest punctured neighbourhood with this property is called the period annulus of the center and it will be denoted by $\mathscr{P}$. Henceforth $\partial \mathscr{P}$ will denote the boundary of $\mathscr{P}$ after embedding it into $\mathbb{R} \mathbb{P}^{2}$. Clearly the center $p$ belongs to $\partial \mathscr{P}$, and in what follows we will call it the inner boundary of the period annulus. We also define the outer boundary of the period annulus to be $\Pi:=\partial \mathscr{P} \backslash\{p\}$. Note that $\Pi$ is a non-empty compact subset of $\mathbb{R P}^{2}$. The period function of the center assigns to each periodic orbits in $\mathscr{P}$ its period. Since the period function is defined on the set of periodic orbits in $\mathscr{P}$, in order to study its qualitative properties usually the first step is to parametrize this set. This can be done by taking an analytic transverse section to the vector field

$$
X=f(x, y) \partial_{x}+g(x, y) \partial_{y}
$$

on $\mathscr{P}$, for instance an orbit of the orthogonal vector field $X^{\perp}$. If $\left\{\gamma_{s}\right\}_{s \in(0,1)}$ is such a parametrization, then $s \longmapsto T(s):=\left\{\right.$ period of $\left.\gamma_{s}\right\}$ is an analytic map that provides the qualitative properties of the period function that we are interested in. In particular the existence of critical periods, which are isolated critical points of this function, i.e. $\hat{s} \in(0,1)$ such that $T^{\prime}(s)=\alpha(s-\hat{s})^{k}+\mathrm{o}\left((s-\hat{s})^{k}\right)$ with $\alpha \neq 0$ and $k \geqslant 1$. In this case we shall say that $\gamma_{\hat{s}}$ is a critical periodic orbit of multiplicity $k$ of the center. One can readily see that this definition does not depend on the particular parametrization of the set of periodic orbits used.

2010 Mathematics Subject Classification. 34C07; 34C23; 34C25.

Key words and phrases: Center, period function, critical periodic orbit, bifurcation, criticality, Chebyshev system.

All the authors are partially supported by the MEC/FEDER grant MTM2014-52209-C2-1-P. D. Rojas is also partially supported by SUR of DEC of Generalitat de Catalunya. 
The study of critical periodic orbits is analogous to the study of limit cycles, which are the main concern of the celebrated Hilbert's 16th problem (see [2,7,24,30] and references there in) and its various weakened versions. Questions related to the behaviour of the period function have been extensively studied by a number of authors. Let us quote for instance the problems of isochronicity (see $[6,12,20]$ ), monotonicity (see $[3,4,26]$ ) or bifurcation of critical periodic orbits (see $[5,25,27]$ ).

Our goal in the present paper is to study the bifurcation of critical periodic orbits from the outer boundary of the period annulus. Of course, as any bifurcation phenomenon, this occurs in case that $X$ depends on a parameter, say $\mu \in \Lambda \subset \mathbb{R}^{d}$. Thus, for each $\mu \in \Lambda$, suppose that $X_{\mu}$ is an analytic vector field on some open set $\mathscr{U}_{\mu} \subset \mathbb{R}^{2}$ with a center at $p_{\mu}$. Following the notation introduced previously, we denote by $\Pi_{\mu}$ the outer boundary of its period annulus $\mathscr{P}_{\mu}$. Concerning the regularity with respect to the parameter, we shall assume that $\left\{X_{\mu}\right\}_{\mu \in \Lambda}$ is a continuous family, meaning that the map $(x, y, \mu) \longmapsto X_{\mu}(x, y)$ is continuous in $\left\{(x, y, \mu) \in \mathbb{R}^{d+2}:(x, y) \in \mathscr{U}_{\mu}, \mu \in \Lambda\right\}$. The present paper is addressed to study of the number of critical periodic orbits of $X_{\mu}$ that can emerge or disappear from $\Pi_{\hat{\mu}}$ as we move slightly the parameter $\mu \approx \hat{\mu}$. We call this number the criticality of the outer boundary and its precise definition is the following, where $d_{H}$ stands for the Hausdorff distance between compact sets of $\mathbb{R P}^{2}$.

Definition 1.1. Consider a continuous family $\left\{X_{\mu}\right\}_{\mu \in \Lambda}$ of planar analytic vector fields with a center and fix some $\hat{\mu} \in \Lambda$. Suppose that the outer boundary of the period annulus varies continuously at $\hat{\mu} \in \Lambda$, meaning that for any $\varepsilon>0$ there exists $\delta>0$ such that $d_{H}\left(\Pi_{\mu}, \Pi_{\hat{\mu}}\right) \leqslant \varepsilon$ for all $\mu \in \Lambda$ with $\|\mu-\hat{\mu}\| \leqslant \delta$. Then, setting

$$
N(\delta, \varepsilon)=\sup \left\{\# \text { critical periodic orbits } \gamma \text { of } X_{\mu} \text { in } \mathscr{P}_{\mu} \text { with } d_{H}\left(\gamma, \Pi_{\hat{\mu}}\right) \leqslant \varepsilon \text { and }\|\mu-\hat{\mu}\| \leqslant \delta\right\},
$$

the criticality of $\left(\Pi_{\hat{\mu}}, X_{\hat{\mu}}\right)$ with respect to the deformation $X_{\mu}$ is $\operatorname{Crit}\left(\left(\Pi_{\hat{\mu}}, X_{\hat{\mu}}\right), X_{\mu}\right):=\inf _{\delta, \varepsilon} N(\delta, \varepsilon)$.

Certainly $\operatorname{Crit}\left(\left(\Pi_{\hat{\mu}}, X_{\hat{\mu}}\right), X_{\mu}\right)$ may be infinite but if it is not, then it gives the maximal number of critical periodic orbits of $X_{\mu}$ that tend to $\Pi_{\hat{\mu}}$ in the Hausdorff sense as $\mu \rightarrow \hat{\mu}$. It is clear that, for a given $\hat{\mu} \in \Lambda$, the geometry of the period annulus $\mathscr{P}_{\hat{\mu}}$ changes as we move $\mu \approx \hat{\mu}$. The assumption that the period annulus varies continuously ensures that these changes do not occur abruptly. In this regard note that $X_{\mu}=-y \partial_{x}+\left(x+\mu x^{3}+x^{5}\right) \partial_{y}$, with $\mu \in \mathbb{R}$, form a continuous family of planar analytic vector fields with a center at the origin for which the outer boundary does not vary continuously at $\mu=2$. Indeed, the period annulus $\mathscr{P}_{\mu}$ is the whole plane for $\mu<2$, whereas is bounded for $\mu=2$ (see [21] for details). Clearly the notion of criticality as defined in Definition 1.1 is meaningless in this situation.

Definition 1.2. We say that $\hat{\mu} \in \Lambda$ is a local regular value of the period function at the outer boundary of the period annulus if $\operatorname{Crit}\left(\left(\Pi_{\hat{\mu}}, X_{\hat{\mu}}\right), X_{\mu}\right)=0$. Otherwise we say that it is a local bifurcation value of the period function at the outer boundary.

In this paper we develop tools that enable to bound the criticality at the outer boundary in case that we deal with a family of potential differential systems. These tools improve the ones that we obtained in [13] although to prove them we shall strongly rely on the results in that paper. In order to set all these results in a context we first recall some well-known facts about the period function of potential differential systems. So consider an analytic function $V$ on some open interval $I$ containing $x=0$ such that $V(0)=V^{\prime}(0)=0$ and $V^{\prime \prime}(0)>0$. Then the potential differential system $X=-y \partial_{x}+V^{\prime}(x) \partial_{y}$ has a non-degenerated center at the origin with the periodic orbits inside the energy levels of the Hamiltonian function $H(x, y)=\frac{1}{2} y^{2}+V(x)$. We have in this case that $H(\mathscr{P})=\left(0, h_{0}\right)$ with $h_{0} \in \mathbb{R}^{+} \cup\{+\infty\}$. Clearly the inner boundary of $\mathscr{P}$, i.e. the center at the origin, is inside the energy level $h=0$. We say, by abuse of language, that the outer boundary $\Pi$ of $\mathscr{P}$ is "inside" the energy level $h=h_{0}$. (We remark that $\Pi$ is a subset of $\mathbb{R P}^{2}$ which may have points outside the vertical strip $I \times \mathbb{R}$, the domain of the Hamiltonian function.) The period $T(h)$ of the periodic orbit $\gamma_{h}$ inside the energy level $\{H(x, y)=h\}$ is given by the Abelian integral

$$
T(h)=\int_{\gamma_{h}} \frac{d x}{y} .
$$

It is well known that $T$ is an analytic function on $\left(0, h_{0}\right)$ that, if the center is non-degenerated, can be extended analytically to $h=0$. Its derivative $T^{\prime}(h)$ is also given by an Abelian integral and we are interested 
in its zeros near $h=h_{0}$, which correspond to critical period orbits near $\Pi$. Suppose now that we deal with a family of potential differential systems $\left\{X_{\mu}\right\}_{\mu \in \Lambda}$ and that the problem is to compute $\operatorname{Crit}\left(\left(\Pi_{\hat{\mu}}, X_{\hat{\mu}}\right), X_{\mu}\right)$ for a given $\hat{\mu} \in \Lambda$. Note firstly that the energy level $h_{0}$ at the outer boundary depends on $\mu$. The tools that we developed in [13] allow to tackle the problem in the following two situations:

- either $h_{0}(\mu)=+\infty$ for all $\mu \approx \hat{\mu}$,

- or $h_{0}(\mu)<+\infty$ for all $\mu \approx \hat{\mu}$.

(We do not treat the case in which in any neighbourhood of $\hat{\mu}$ there are $\mu_{1}$ and $\mu_{2}$ with $h\left(\mu_{1}\right)=+\infty$ and $h\left(\mu_{2}\right)<+\infty$.) For each one of these two situations, we gave a theoretical result for $\operatorname{Crit}\left(\left(\Pi_{\hat{\mu}}, X_{\hat{\mu}}\right), X_{\mu}\right)=0$ and another one for $\operatorname{Crit}\left(\left(\Pi_{\hat{\mu}}, X_{\hat{\mu}}\right), X_{\mu}\right) \leqslant 1$. It is to be noted that the approach we followed to prove those results, as well as their assumptions, are very different for the cases $h_{0}=+\infty$ and $h_{0}<+\infty$. In this paper we go further and prove Theorems A and B, addressed to cases $h_{0}=+\infty$ and $h_{0}<+\infty$, respectively, in which we give sufficient conditions in order that $\operatorname{Crit}\left(\left(\Pi_{\hat{\mu}}, X_{\hat{\mu}}\right), X_{\mu}\right) \leqslant n$ for $n \in \mathbb{N} \cup\{0\}$. The idea in both cases is to find functions $\phi_{\mu}^{i}(h), i=1,2, \ldots, n$, verifying that there exist $\delta, \varepsilon>0$ such that if $\|\mu-\hat{\mu}\|<\delta$, then $\left(\phi_{\mu}^{1}, \phi_{\mu}^{2}, \ldots, \phi_{\mu}^{n}, T_{\mu}^{\prime}\right)$ is an extended complete Chebyshev system (ECT-system for short, see Definition 2.1) on the interval $\left(h_{0}(\mu)-\varepsilon, h_{0}(\mu)\right)$. This implies in particular that $T_{\mu}^{\prime}(h)$ has at most $n$ zeros for $h \in\left(h_{0}(\mu)-\varepsilon, h_{0}(\mu)\right)$, counted with multiplicities, for all $\mu \approx \hat{\mu}$ and, accordingly, $\operatorname{Crit}\left(\left(\Pi_{\hat{\mu}}, X_{\hat{\mu}}\right), X_{\mu}\right) \leqslant n$. We choose different type of functions $\phi_{\mu}^{i}$ for the cases $h_{0}=+\infty$ and $h_{0}<+\infty$, but in both situations we take them simple enough in order that $\left(\phi_{\mu}^{1}, \phi_{\mu}^{2}, \ldots, \phi_{\mu}^{n}\right)$ is an ECT-system on $\left(0, h_{0}(\mu)\right)$. Taking this into account, the problem is then to guarantee that the Wronskian (see Definition 2.2) of $\left(\phi_{\mu}^{1}, \phi_{\mu}^{2}, \ldots, \phi_{\mu}^{n}, T_{\mu}^{\prime}\right)$ is non-vanishing near $h=h_{0}(\mu)$ for all $\mu \approx \hat{\mu}$.

Theorems A and B can be compared to the results obtained in the series of papers [16-19] by Mardešić et al. because both studies deal with the bifurcation of critical periodic orbits from the outer boundary of the period annulus. However striking differences exist. The first one is that their results apply to differential systems which need not be potential, but on the other hand their approach requires that the differential system has a meromorphic extension to $\Pi$ (for instance, starting with a polynomial system and making its Poincaré compactification). The second one is due to the fact that we bound the criticality by embedding the derivative of the period function in an ECT-system, whereas their approach is to obtain the asymptotic expansion of the period function near the outer boundary and then compute the coefficient of the principal term. The testing ground for the results by Mardešic et al. is the family of Loud's centers. In this regard it is to be pointed out that the Loud's family can be brought to potential form by means of an explicit coordinate transformation, see [28, Lemma 2.2], and hence it is susceptible to be studied with our techniques. We expect to exploit this in a forthcoming paper that we hope will prove some aspects of the conjectural bifurcation diagram of the period function of the Loud's centers proposed in [18].

Our testing ground is the two-parametric family of potential differential systems given by

$$
\left\{\begin{array}{l}
\dot{x}=-y \\
\dot{y}=(x+1)^{p}-(x+1)^{q},
\end{array}\right.
$$

which has a non-degenerated center at the origin for all $\mu:=(q, p)$ varying inside $\Lambda:=\left\{(q, p) \in \mathbb{R}^{2}: p>q\right\}$. Note that, for each $\mu \in \Lambda, X_{\mu}:=-y \partial_{x}+\left((x+1)^{p}-(x+1)^{q}\right) \partial_{y}$ is analytic on $\left\{(x, y) \in \mathbb{R}^{2}: x>-1\right\}$. We became interested in this family because of the previous results by Miyamoto and Yagasaki in [22] concerning the monotonicity of the period function for $q=1$ and $p \in \mathbb{N}$. Later Yagasaki improved the result showing in [29] the monotonicity of the period function for $q=1$ and any real number $p>1$. We studied afterwards the whole family $\left\{X_{\mu}\right\}_{\mu \in \Lambda}$ in $[13,14]$. To be more precise, in [14] we were concerned with the monotonicity of the period function, the criticality of the inner boundary and the criticality of the interior of the period annulus of the isochronous centers. In [13] we studied the criticality of the outer boundary and it is precisely the result we obtained there for the family (1) the one that we seek to improve here. In short, see Figure 1, we proved that $\operatorname{Crit}\left(\left(\Pi_{\hat{\mu}}, X_{\hat{\mu}}\right), X_{\mu}\right)=0$ if $\hat{\mu} \in \Lambda \backslash\left\{\Gamma_{B} \cup \Gamma_{U}\right\}$ and $\operatorname{Crit}\left(\left(\Pi_{\hat{\mu}}, X_{\hat{\mu}}\right), X_{\mu}\right) \geqslant 1$ if $\hat{\mu} \in \Gamma_{B}$. Without going into detail for the sake of shortness, we proved moreover that the criticality is exactly one for parameters inside two segments in $\Gamma_{B}$. By applying the general tools developed in this paper we can go further and prove the following result, where $f(p):=(2+2 p)^{\frac{2+2 p}{1+2 p}}-2(1+2 p)$. 




Figure 1: Bifurcation diagram of the period function of the family (1) at the outer boundary of the period annulus according to [13, Theorem E], where $\Gamma_{B}$ and $\Gamma_{U}$ stand, respectively, for the union of the solid and dotted lines. Then $\operatorname{Crit}\left(\left(\Pi_{\hat{\mu}}, X_{\hat{\mu}}\right), X_{\mu}\right)=0$ if $\hat{\mu} \in \Lambda \backslash\left\{\Gamma_{B} \cup \Gamma_{U}\right\}$ and $\operatorname{Crit}\left(\left(\Pi_{\hat{\mu}}, X_{\hat{\mu}}\right), X_{\mu}\right) \geqslant 1$ if $\hat{\mu} \in \Gamma_{B}$.

Theorem C. Let $\left\{X_{\mu}\right\}_{\mu \in \Lambda}$ be the family of potential vector fields in (1) and consider the period function of the center at the origin. Then the following hold:

(a) If $\hat{\mu}=\left(-\frac{1}{2}, \hat{p}\right)$ with $\hat{p} \in\left(-\frac{1}{2},+\infty\right) \backslash\left\{0, p_{0}\right\}$, where $p_{0} \approx 1.20175$ is the unique zero of $f$ on $\left(-\frac{1}{2},+\infty\right)$, then $\operatorname{Crit}\left(\left(\Pi_{\hat{\mu}}, X_{\hat{\mu}}\right), X_{\mu}\right)=0$.

(b) If $\hat{\mu}=(0, \hat{p})$ with $\hat{p} \in(0,+\infty) \backslash\left\{\frac{1}{2}, 1\right\}$, then $\operatorname{Crit}\left(\left(\Pi_{\hat{\mu}}, X_{\hat{\mu}}\right), X_{\mu}\right)=1$.

It is worth mentioning that the centers corresponding to the parameters $\mu=\left(-\frac{1}{2}, 0\right)$ and $\mu=(0,1)$ are isochronous. In this regard the result in $(a)$ and $(b)$ is optimal for these particular parameters.

The paper has three additional sections organized in the following way. In Section 2 we introduce the notions of ECT-system and Wronskian and recall well-known properties that relate them. Furthermore we obtain the analytical tools that we shall later use to prove the results about the criticality, which is done in Section 3. To be more precise, we treat the case $h_{0}=+\infty$ and prove Theorem A in Section 3.1, whereas we consider the case $h_{0}<+\infty$ and show Theorem B in Section 3.2. Finally, to illustrate its applicability we prove Theorem $\mathrm{C}$ in Section 4.

\section{Previous analytic results}

This section is devoted to obtain the technical tools that we shall later use to prove the results concerning the criticality. To this end let us take $a \in \mathbb{R}^{+} \cup\{+\infty\}$ and consider the integral operator

$$
\mathscr{F}: \mathscr{C}^{\omega}([0, a)) \longrightarrow \mathscr{C}^{\omega}([0, a))
$$

defined by

$$
\mathscr{F}[f](x):=\int_{0}^{\frac{\pi}{2}} f(x \sin \theta) d \theta .
$$

Here, and in what follows, $\mathscr{C}^{\omega}([0, a))$ stands for the set of analytic functions on $(0, a)$ that can be analytically extended to $x=0$. The reason why we are interested in this operator is because we can relate it with the derivative of the period function of $X=-y \partial_{x}+V^{\prime}(x) \partial_{y}$. Indeed, it is well known (see for instance [3, 15]) that the period $T(h)$ of the periodic orbit $\gamma_{h}$ inside the energy level $\left\{\frac{1}{2} y^{2}+V(x)=h\right\}$ is given by

$$
T(h)=\int_{\gamma_{h}} \frac{d x}{y}=\sqrt{2} \int_{-\frac{\pi}{2}}^{\frac{\pi}{2}}\left(g^{-1}\right)^{\prime}(\sqrt{h} \sin \theta) d \theta,
$$


where $g(x)=\operatorname{sgn}(x) \sqrt{V(x)}$. Consequently

$$
T^{\prime}(h)=\frac{1}{\sqrt{2 h}} \int_{-\frac{\pi}{2}}^{\frac{\pi}{2}}\left(g^{-1}\right)^{\prime \prime}(\sqrt{h} \sin \theta) \sin \theta d \theta,
$$

so that $\sqrt{2} h^{2} T^{\prime}\left(h^{2}\right)=\mathscr{F}[f](h)$ with $f(x)=x\left(g^{-1}\right)^{\prime \prime}(x)-x\left(g^{-1}\right)^{\prime \prime}(-x)$.

Next we recall the notions of Chebyshev system and Wronskian, that will be very useful for our purposes.

Definition 2.1. Let $f_{0}, f_{1}, \ldots f_{n-1}$ be analytic functions on an open real interval $I$. The ordered set $\left(f_{0}, f_{1}, \ldots f_{n-1}\right)$ is an extended complete Chebyshev system (for short, a ECT-system) on $I$ if, for all $k=$ $1,2, \ldots n$, any nontrivial linear combination

$$
\alpha_{0} f_{0}(x)+\alpha_{1} f_{1}(x)+\cdots+\alpha_{k-1} f_{k-1}(x)
$$

has at most $k-1$ isolated zeros on $I$ counted with multiplicities. (Let us mention that, in these abbreviations, "T" stands for Tchebycheff, which in some sources is the transcription of the Russian name Chebyshev).

Definition 2.2. Let $f_{0}, f_{1}, \ldots, f_{k-1}$ be analytic functions on an open interval $I$ of $\mathbb{R}$. Then

$$
W\left[f_{0}, f_{1}, \ldots, f_{k-1}\right](x)=\operatorname{det}\left(f_{j}^{(i)}(x)\right)_{0 \leqslant i, j \leqslant k-1}=\left|\begin{array}{ccc}
f_{0}(x) & \cdots & f_{k-1}(x) \\
f_{0}^{\prime}(x) & \cdots & f_{k-1}^{\prime}(x) \\
& \vdots & \\
f_{0}^{(k-1)}(x) & \cdots & f_{k-1}^{(k-1)}(x)
\end{array}\right|
$$

is the Wronskian of $\left(f_{0}, f_{1}, \ldots, f_{k-1}\right)$ at $x \in I$.

These two notions are closely related by the following well-known result (see for instance [11]).

Lemma 2.3. $\left(f_{0}, f_{1}, \ldots, f_{n-1}\right)$ is an ECT-system on $I$ if and only if, for each $k=1,2, \ldots, n$,

$$
W\left[f_{0}, f_{1}, \ldots, f_{k-1}\right](x) \neq 0 \text { for all } x \in I .
$$

Our goal is to complete $\mathscr{F}[f]$ with some analytic functions $g_{0}, \ldots, g_{n-1}$ in order that $\left(g_{0}, \ldots, g_{n-1}, \mathscr{F}[f]\right)$ form an ECT-system on $(a-\varepsilon, a)$ for some $\varepsilon>0$. In particular this will imply that $\mathscr{F}[f](x)$, and so the derivative of the period function, has at most $n$ isolated zeroes for $x \approx a$ counted with multiplicities. Then to obtain the desired upper bounds on the criticality, the delicate point will be as usual to guarantee the uniformity with respect to the parameters of the system. Thus we aim to find sufficient conditions in terms of $f$ in order that $\mathscr{F}[f]$ can be embedded into an ECT-system. These conditions will be formulated using the notions that we introduce next:

Definition 2.4. Let $f$ be an analytic function on $I=(a, b)$. We say that $f$ is quantifiable at $b$ by $\alpha$ with limit $\ell$ in case that:

(i) If $b \in \mathbb{R}$, then $\lim _{x \rightarrow b^{-}} f(x)(b-x)^{\alpha}=\ell$ and $\ell \neq 0$.

(ii) If $b=+\infty$, then $\lim _{x \rightarrow+\infty} \frac{f(x)}{x^{\alpha}}=\ell$ and $\ell \neq 0$.

We call $\alpha$ the quantifier of $f$ at $b$. We shall use the analogous definition at $a$.

Definition 2.5. Let $\Lambda$ be an open subset of $\mathbb{R}^{d}$ and suppose that, for each $\mu \in \Lambda, f_{\mu}$ is an analytic function on some real interval $I_{\mu}$. Suppose furthermore that the map $(x, \mu) \longmapsto f_{\mu}(x)$ is continuous on $\left\{(x, \mu) \in \mathbb{R} \times \Lambda: x \in I_{\mu}\right\}$. Then we say that $\left\{f_{\mu}\right\}_{\mu \in \Lambda}$ is a continuous family of analytic functions on $I_{\mu}$.

Definition 2.6. Let $\left\{f_{\mu}\right\}_{\mu \in \Lambda}$ be a continuous family of analytic functions on $I_{\mu}=(a(\mu), b(\mu))$. Assume that $b$ is either a continuous function from $\Lambda$ to $\mathbb{R}$ or $b(\mu)=+\infty$ for all $\mu \in \Lambda$. Given $\hat{\mu} \in \Lambda$ we shall say that $\left\{f_{\mu}\right\}_{\mu \in \Lambda}$ is continuously quantifiable in $\hat{\mu}$ at $b(\mu)$ by $\alpha(\mu)$ with limit $\ell$ if there exists an open neighbourhood $U$ of $\hat{\mu}$ such that $f_{\mu}$ is quantifiable at $b(\mu)$ by $\alpha(\mu)$ for all $\mu \in U$ and, moreover, 
(i) In case that $b(\hat{\mu})<+\infty$, then $\lim _{(x, \mu) \rightarrow(b(\hat{\mu}), \hat{\mu})} f_{\mu}(x)(b(\mu)-x)^{\alpha(\mu)}=\ell$ and $\ell \neq 0$.

(ii) In case that $b(\hat{\mu})=+\infty$, then $\lim _{(x, \mu) \rightarrow(+\infty, \hat{\mu})} \frac{f_{\mu}(x)}{x^{\alpha(\mu)}}=\ell$ and $\ell \neq 0$.

We shall use the analogous definition for the left endpoint of $I_{\mu}$.

Remark 2.7. Notice that the map $\alpha: U \longrightarrow \mathbb{R}$ that appears in the previous definition must be continuous at $\hat{\mu}$, otherwise there exists a sequence $\left\{\mu_{n}\right\}_{n \in \mathbb{N}}$ such that $\lim _{n \rightarrow \infty} \alpha\left(\mu_{n}\right)=\alpha(\hat{\mu})+\kappa$ with $\kappa \neq 0$. Then, for instance in case that $b(\hat{\mu})=+\infty$, we would have

$$
\ell=\lim _{(x, n) \rightarrow(+\infty,+\infty)} \frac{f_{\mu_{n}}(x)}{x^{\alpha\left(\mu_{n}\right)}}=\lim _{x \rightarrow+\infty}\left(\lim _{n \rightarrow+\infty} \frac{f_{\mu_{n}}(x)}{x^{\alpha\left(\mu_{n}\right)}}\right)=\lim _{x \rightarrow+\infty} \frac{f_{\hat{\mu}}(x)}{x^{\alpha(\hat{\mu})+\kappa}}
$$

which, on account of $\ell \neq 0$, contradicts that $\lim _{x \rightarrow+\infty} \frac{f_{\hat{\mu}}(x)}{x^{\alpha(\hat{\mu})}}$ is finite and different from zero by definition.

Lemma 2.8. Let $\left\{f_{\mu}\right\}_{\mu \in \Lambda}$ be a continuous family of analytic functions on $[0,+\infty)$. Assume that $\left\{f_{\mu}\right\}_{\mu \in \Lambda}$ and $\left\{f_{\mu}^{\prime}\right\}_{\mu \in \Lambda}$ are continuously quantifiable in $\hat{\mu}$ at $+\infty$ by $\alpha(\mu)$ and $\beta(\mu)$ with limit a and b, respectively, and that $\alpha(\hat{\mu}) \neq 0$. Then $\beta=\alpha-1$ and $b=\alpha(\hat{\mu}) a$.

Proof. The result follows by using Hôpital's Rule and the uniqueness of the quantifier (see Remark 2.7).

Definition 2.9. Let $f$ be an analytic function on $[0,+\infty)$. Then, for each $n \in \mathbb{N}$, we call

$$
M_{n}[f]:=\int_{0}^{+\infty} x^{2 n-2} f(x) d x
$$

the $n$-th momentum of $f$, whenever it is well defined.

Following the previous definitions and notation, the next result gathers Theorems 2.13 and 2.17 in [13].

Theorem 2.10. Let $\Lambda$ be an open subset of $\mathbb{R}^{d}$ and consider a continuous family $\left\{f_{\mu}\right\}_{\mu \in \Lambda}$ of analytic functions on $[0,+\infty)$. Suppose that $\left\{f_{\mu}\right\}_{\mu \in \Lambda}$ is continuously quantifiable in $\Lambda$ at $+\infty$ by $\alpha(\mu)$. The following assertions hold:

(a) If $\alpha(\hat{\mu})>-1$, then $\left\{\mathscr{F}\left[f_{\mu}\right]\right\}_{\mu \in \Lambda}$ is continuously quantifiable in $\hat{\mu}$ at $+\infty$ by $\alpha(\mu)$.

(b) If $\alpha(\hat{\mu})<-1$, let us take $m \in \mathbb{N}$ such that $\alpha(\hat{\mu})+2 m \in[-1,1)$. In this case:

(b1) If $M_{1}\left[f_{\mu}\right] \equiv M_{2}\left[f_{\mu}\right] \equiv \ldots \equiv M_{j-1}\left[f_{\mu}\right] \equiv 0$ and $M_{j}\left[f_{\hat{\mu}}\right] \neq 0$ for some $1 \leqslant j \leqslant m$, then $\left\{\mathscr{F}\left[f_{\mu}\right]\right\}_{\mu \in \Lambda}$ is continuously quantifiable in $\hat{\mu}$ at $+\infty$ by $1-2 j$.

(b2) If $M_{1}\left[f_{\mu}\right] \equiv M_{2}\left[f_{\mu}\right] \equiv \cdots \equiv M_{m}\left[f_{\mu}\right] \equiv 0$ and $\alpha(\hat{\mu})+2 m \notin\{-1,0\}$, then $\left\{\mathscr{F}\left[f_{\mu}\right]\right\}_{\mu \in \Lambda}$ is continuously quantifiable in $\hat{\mu}$ at $+\infty$ by $\alpha(\mu)$.

We point out that the hypothesis $M_{1}\left[f_{\mu}\right] \equiv M_{2}\left[f_{\mu}\right] \equiv \ldots \equiv M_{j-1}\left[f_{\mu}\right] \equiv 0$ in the previous statement is void for $j=1$.

Given $\nu_{1}, \nu_{2}, \ldots, \nu_{n} \in \mathbb{R}$, we define the linear ordinary differential operator

$$
\mathscr{L}_{\boldsymbol{\nu}_{n}}: \mathscr{C}^{\omega}((0,+\infty)) \longrightarrow \mathscr{C}^{\omega}((0,+\infty))
$$

given by

$$
\mathscr{L}_{\boldsymbol{\nu}_{n}}[f](x):=\frac{W\left[x^{\nu_{1}}, x^{\nu_{2}}, \ldots, x^{\nu_{n}}, f(x)\right]}{x^{\sum_{i=1}^{n}\left(\nu_{i}-i\right)}} .
$$

Here, and in what follows, for the sake of shortness we use the notation $\boldsymbol{\nu}_{n}=\left(\nu_{1}, \ldots, \nu_{n}\right)$. Furthermore we define $\mathscr{L}_{\boldsymbol{\nu}_{0}}=i d$ in order that the statements of the next results contemplate the case $n=0$ as well.

The rest of the present section is devoted to study under which conditions the quantifier of $\mathscr{L}_{\boldsymbol{\nu}_{n}}\left[f_{\mu}\right]$ at $x=+\infty$ enables to quantify $\left(\mathscr{L}_{\boldsymbol{\nu}_{n}} \circ \mathscr{F}\right)\left[f_{\mu}\right]$ at $x=+\infty$. To this end some previous technical results about Wronskians are needed. The first two lemmas are well known (see, respectively, [15] and $[10,23])$. 
Lemma 2.11. Let $f_{0}, f_{1}, \ldots, f_{n-1}$ be analytic functions. Then the following statements hold:

(a) $W\left[f_{0} \circ \varphi, \ldots, f_{n-1} \circ \varphi\right](x)=\left(\varphi^{\prime}(x)\right)^{\frac{(n-1) n}{2}} W\left[f_{0}, \ldots, f_{n-1}\right](\varphi(x))$ for any analytic diffeomorphism $\varphi$.

(b) $W\left[g f_{0}, \ldots, g f_{n-1}\right](x)=g(x)^{n} W\left[f_{0}, \ldots, f_{n-1}\right](x)$ for any analytic function $g$.

Lemma 2.12. Let $f_{0}, f_{1}, \ldots, f_{n}$ be analytic functions on an open interval $I$ such that $W\left[f_{0}, \ldots, f_{n-2}, f_{n-1}\right]$ does not vanish on $I$. Then

$$
\left(\frac{W\left[f_{0}, \ldots, f_{n-2}, f_{n}\right]}{W\left[f_{0}, \ldots, f_{n-2}, f_{n-1}\right]}\right)^{\prime}=\frac{W\left[f_{0}, \ldots, f_{n}\right] W\left[f_{0}, \ldots, f_{n-2}\right]}{\left(W\left[f_{0}, \ldots, f_{n-2}, f_{n-1}\right]\right)^{2}} .
$$

Lemma 2.13. Given $\nu_{1}, \nu_{2}, \ldots, \nu_{n} \in \mathbb{R}$, the following identity holds:

$$
\frac{W\left[x^{\nu_{1}}, x^{\nu_{2}}, \ldots, x^{\nu_{n}}\right]}{x^{\sum_{i=1}^{n}\left(\nu_{i}-i\right)}}=x^{n} \prod_{\substack{i, j=1 \\ i>j}}^{n}\left(\nu_{i}-\nu_{j}\right) .
$$

Proof. We prove the result by induction on $n$. Since the base case $n=1$ is obvious, let us show the induction step. By applying $(b)$ in Lemma 2.11 we get

$$
W\left[x^{\nu_{1}}, \ldots, x^{\nu_{n-1}}, x^{\nu_{n}}\right]=x^{n \nu_{n}} W\left[x^{\nu_{1}-\nu_{n}}, \ldots, x^{\nu_{n-1}-\nu_{n}}, 1\right] .
$$

Let us denote $\beta_{i}:=\nu_{i}-\nu_{n}$ for shortness. Then, using well-known properties of the determinant and the induction hypothesis,

$$
\begin{aligned}
W\left[x^{\beta_{1}}, \ldots, x^{\beta_{n-1}}, 1\right] & =(-1)^{n-1} W\left[\beta_{1} x^{\beta_{1}-1}, \ldots, \beta_{n-1} x^{\beta_{n-1}-1}\right]=(-1)^{n-1} W\left[x^{\beta_{1}-1}, \ldots, x^{\beta_{n-1}-1}\right] \prod_{k=1}^{n-1} \beta_{k} \\
& =(-1)^{n-1} x^{\sum_{i=1}^{n-1}\left(\beta_{i}-1-i\right)} x^{n-1} \prod_{k=1}^{n-1} \beta_{k} \prod_{\substack{i, j=1 \\
i>j}}^{n-1}\left(\beta_{i}-\beta_{j}\right) .
\end{aligned}
$$

Consequently, substituting the previous equality in (5), we have

$$
\begin{aligned}
W\left[x^{\nu_{1}}, \ldots, x^{\nu_{n-1}}, x^{\nu_{n}}\right] & =x^{n \nu_{n}}(-1)^{n-1} x^{\sum_{i=1}^{n-1}\left(\nu_{i}-\nu_{n}-1-i\right)} x^{n-1} \prod_{i=k}^{n-1}\left(\nu_{k}-\nu_{n}\right) \prod_{\substack{i, j=1 \\
i>j}}^{n-1}\left(\nu_{i}-\nu_{j}\right) \\
& =x^{n} x^{\sum_{i=1}^{n}\left(\nu_{i}-i\right)} \prod_{\substack{i, j=1 \\
i>j}}^{n}\left(\nu_{i}-\nu_{j}\right),
\end{aligned}
$$

where we used $\beta_{i}=\nu_{i}-\nu_{n}$ in the first equality and the second one follows by means of some easy manipulations. This shows the induction step and so the result is proved.

The previous lemma enables to write the differential operator under consideration as a quotient of Wronskians. Indeed, if $\nu_{1}, \nu_{2}, \ldots, \nu_{n}$ are pairwise distinct, then we have that

$$
\mathscr{L}_{\boldsymbol{\nu}_{n}}[f](x)=x^{n} \prod_{\substack{i, j=1 \\ i>j}}^{n}\left(\nu_{i}-\nu_{j}\right) \frac{W\left[x^{\nu_{1}}, \ldots, x^{\nu_{n}}, f(x)\right]}{W\left[x^{\nu_{1}}, \ldots, x^{\nu_{n}}\right]} .
$$

At this point it is worth noting that the linear ordinary differential operator

$$
f \longmapsto \frac{W\left[\phi_{1}, \phi_{2}, \ldots, \phi_{n}, f\right]}{W\left[\phi_{1}, \phi_{2}, \ldots, \phi_{n}\right]}
$$


has already appeared in the literature in relation with the so called "Chebyshev asymptotic scales" (see [8,9] and references therein). Of course, it is also related to the division-derivation algorithm (see [24] for instance) due to the fact that its kernel is spanned by $\left\{\phi_{1}, \phi_{2}, \ldots, \phi_{n}\right\}$.

Our next result shows that the integral operator $\mathscr{F}$ and the differential operator $\mathscr{L}_{\boldsymbol{\nu}_{n}}$ commute. This fact is the key point in order to prove our main results.

Proposition 2.14. For any given $f \in \mathscr{C}^{\omega}((0,+\infty))$ and $\nu_{1}, \ldots, \nu_{n} \in \mathbb{R}$, the following recurrence holds:

$$
\mathscr{L}_{\boldsymbol{\nu}_{n}}[f](x)=c_{n}\left(x \mathscr{L}_{\boldsymbol{\nu}_{n-1}}[f]^{\prime}(x)-\nu_{n} \mathscr{L}_{\boldsymbol{\nu}_{n-1}}[f](x)\right),
$$

where $c_{1}:=1$ and $c_{n}:=\prod_{i=1}^{n-1}\left(\nu_{n}-\nu_{i}\right)$ for $n \geqslant 2$. In particular, if $f$ can be extended analytically to $x=0$, then $\mathscr{L}_{\boldsymbol{\nu}_{n}}[f]$ can be extended analytically to $x=0$. Finally, $\mathscr{F} \circ \mathscr{L}_{\boldsymbol{\nu}_{n}}=\mathscr{L}_{\boldsymbol{\nu}_{n}} \circ \mathscr{F}$.

Proof. We can suppose that $\nu_{1}, \nu_{2}, \ldots, \nu_{n}$ are pairwise distinct, otherwise there is nothing to be proved. The case $n=1$ of the recurrence is straightforward because, by definition, $\mathscr{L}_{\boldsymbol{\nu}_{0}}=i d$ and

$$
\mathscr{L}_{\boldsymbol{\nu}_{1}}[f](x)=\frac{W\left[x^{\nu_{1}}, f(x)\right]}{x^{\nu_{1}-1}}=x f^{\prime}(x)-\nu_{1} f(x) .
$$

Let us show now the case $n \geqslant 2$. To this end take any $k \in\{1,2, \ldots, n-1\}$ and note that, by Lemma 2.12 ,

$$
\left(\frac{W\left[x^{\nu_{1}}, \ldots, x^{\nu_{k}}, f(x)\right]}{W\left[x^{\nu_{1}}, \ldots, x^{\nu_{k+1}}\right]}\right)^{\prime}=\frac{W\left[x^{\nu_{1}}, \ldots, x^{\nu_{k+1}}, f(x)\right] W\left[x^{\nu_{1}}, \ldots, x^{\nu_{k}}\right]}{\left(W\left[x^{\nu_{1}}, \ldots, x^{\nu_{k+1}}\right]\right)^{2}} .
$$

Hence, some easy computations taking Lemma 2.13 into account show that

$$
W\left[x^{\nu_{1}}, \ldots, x^{\nu_{k}}, f(x)\right]^{\prime}=\frac{W\left[x^{\nu_{1}}, \ldots, x^{\nu_{k+1}}, f(x)\right]}{x^{\nu_{k+1}-k} \prod_{i=1}^{k}\left(\nu_{k+1}-\nu_{i}\right)}+\frac{1}{x}\left(\nu_{k+1}+\sum_{i=1}^{k}\left(\nu_{i}-i\right)\right) W\left[x^{\nu_{1}}, \ldots, x^{\nu_{k}}, f(x)\right] .
$$

By definition, see (4), we have on the other hand that

$$
W\left[x^{\nu_{1}}, x^{\nu_{2}}, \ldots, x^{\nu_{k}}, f(x)\right]^{\prime}=x^{\sum_{i=1}^{k}\left(\nu_{i}-i\right)} \mathscr{L}_{\boldsymbol{\nu}_{k}}[f]^{\prime}(x)+\frac{1}{x} \sum_{i=1}^{k}\left(\nu_{i}-i\right) W\left[x^{\nu_{1}}, x^{\nu_{2}}, \ldots, x^{\nu_{k}}, f(x)\right] .
$$

Then, using (6) and the above equality, after some computations we get

$$
\mathscr{L}_{\boldsymbol{\nu}_{k+1}}[f](x)=\prod_{i=1}^{k}\left(\nu_{k+1}-\nu_{i}\right)\left(x \mathscr{L}_{\boldsymbol{\nu}_{k}}[f]^{\prime}(x)-\nu_{k+1} \mathscr{L}_{\boldsymbol{\nu}_{k}}[f](x)\right) .
$$

Thus, taking $k=n-1$ we obtain the recurrence in the statement for $n \geqslant 2$.

Let us turn to the proof of $\mathscr{F} \circ \mathscr{L}_{\boldsymbol{\nu}_{n}}=\mathscr{L}_{\boldsymbol{\nu}_{n}} \circ \mathscr{F}$. We show it by induction on $n \geqslant 0$ taking advantage of the recurrence we have just proved. The base case $n=0$ is clear because $\mathscr{L}_{\boldsymbol{\nu}_{0}}=i d$. To show the induction step take any $g \in \mathscr{C}^{\omega}((0,+\infty))$ and note that $\mathscr{F}[g]^{\prime}(x)=\frac{1}{x} \mathscr{F}\left[x g^{\prime}(x)\right]$. Thus, deriving the induction hypothesis, we get $0=\mathscr{F}\left[\mathscr{L}_{\boldsymbol{\nu}_{n}}[g]\right]^{\prime}(x)-\mathscr{L}_{\boldsymbol{\nu}_{n}}[\mathscr{F}[g]]^{\prime}(x)=\frac{1}{x} \mathscr{F}\left[x \mathscr{L}_{\boldsymbol{\nu}_{n}}[g]^{\prime}(x)\right]-\mathscr{L}_{\boldsymbol{\nu}_{n}}[\mathscr{F}[g]]^{\prime}(x)$. Therefore,

$$
\begin{aligned}
0 & =\mathscr{F}\left[x \mathscr{L}_{\boldsymbol{\nu}_{n}}[g]^{\prime}(x)\right]-x \mathscr{L}_{\boldsymbol{\nu}_{n}}[\mathscr{F}[g]]^{\prime}(x) \\
& =\mathscr{F}\left[\frac{1}{c_{n+1}} \mathscr{L}_{\boldsymbol{\nu}_{n+1}}[g]+\nu_{n+1} \mathscr{L}_{\boldsymbol{\nu}_{n}}[g]\right](x)-\left(\frac{1}{c_{n+1}} \mathscr{L}_{\boldsymbol{\nu}_{n+1}}[\mathscr{F}[g]]+\nu_{n+1} \mathscr{L}_{\boldsymbol{\nu}_{n}}[\mathscr{F}[g]]\right)(x) \\
& =\frac{1}{c_{n+1}} \mathscr{F}\left[\mathscr{L}_{\boldsymbol{\nu}_{n+1}}[g]\right](x)+\nu_{n+1} \mathscr{F}\left[\mathscr{L}_{\boldsymbol{\nu}_{n}}[g]\right](x)-\frac{1}{c_{n+1}} \mathscr{L}_{\boldsymbol{\nu}_{n+1}}[\mathscr{F}[g]](x)-\nu_{n+1} \mathscr{L}_{\boldsymbol{\nu}_{n}}[\mathscr{F}[g]](x) \\
& =\frac{1}{c_{n+1}}\left(\mathscr{F}\left[\mathscr{L}_{\boldsymbol{\nu}_{n+1}}[g]\right](x)-\mathscr{L}_{\boldsymbol{\nu}_{n+1}}[\mathscr{F}[g]](x)\right),
\end{aligned}
$$

where in the second equality we use twice the recurrence, taking $f=g$ and $f=\mathscr{F}[g]$, in the third one the linearity of $\mathscr{F}$, and in the fourth one the induction hypothesis. Hence $\mathscr{F}\left[\mathscr{L}_{\boldsymbol{\nu}_{n+1}}[g]\right]-\mathscr{L}_{\boldsymbol{\nu}_{n+1}}[\mathscr{F}[g]]=0$ and so the induction step follows. This concludes the proof of the result. 
Lemma 2.15. Let $f$ be an analytic function on $[0,+\infty), \nu_{1}, \nu_{2}, \ldots, \nu_{n} \in \mathbb{R}$ and $\ell \in \mathbb{N}$. Let us assume that $\mathscr{L}_{\boldsymbol{\nu}_{n-1}}[f]$ is quantifiable at $+\infty$ by $\xi$. If $\xi<1-2 \ell$, then

$$
M_{\ell}\left[\mathscr{L}_{\boldsymbol{\nu}_{n}}[f]\right]=c_{n}\left(1-2 \ell-\nu_{n}\right) M_{\ell}\left[\mathscr{L}_{\boldsymbol{\nu}_{n-1}}[f]\right]
$$

where $c_{1}:=1$ and $c_{n}:=\prod_{i=1}^{n-1}\left(\nu_{n}-\nu_{i}\right)$ for $n \geqslant 2$.

Proof. By using the recurrence in Proposition 2.14 and the definition of the momentum,

$$
M_{\ell}\left[\mathscr{L}_{\boldsymbol{\nu}_{n}}[f]\right]=\int_{0}^{+\infty} x^{2 \ell-2} \mathscr{L}_{\boldsymbol{\nu}_{n}}[f](x) d x=c_{n} \int_{0}^{+\infty} x^{2 \ell-2}\left(x \mathscr{L}_{\boldsymbol{\nu}_{n-1}}[f]^{\prime}(x)-\nu_{n} \mathscr{L}_{\boldsymbol{\nu}_{n-1}}[f](x)\right) d x .
$$

Since $\mathscr{L}_{\boldsymbol{\nu}_{n-1}}[f]$ is quantifiable at infinity by $\xi<1-2 \ell$, we can assert that $\lim _{x \rightarrow+\infty} x^{2 \ell-1} \mathscr{L}_{\boldsymbol{\nu}_{n-1}}[f](x)=0$. Moreover, by Proposition $2.14, \mathscr{L}_{\boldsymbol{\nu}_{n-1}}[f]$ is analytic at $x=0$. So integrating by parts we get

$$
M_{\ell}\left[\mathscr{L}_{\boldsymbol{\nu}_{n}}[f]\right]=c_{n}\left(1-2 \ell-\nu_{n}\right) \int_{0}^{+\infty} x^{2 \ell-2} \mathscr{L}_{\boldsymbol{\nu}_{n-1}}[f](x) d x=c_{n}\left(1-2 \ell-\nu_{n}\right) M_{\ell}\left[\mathscr{L}_{\boldsymbol{\nu}_{n-1}}[f]\right]
$$

and this proves the result.

In the following statement $\nu_{1}, \nu_{2}, \ldots, \nu_{n}$ are not real numbers any more but continuous functions on $\Lambda$. For shortness, we keep using the notation $\boldsymbol{\nu}_{n}(\mu)=\left(\nu_{1}(\mu), \ldots, \nu_{n}(\mu)\right)$.

Proposition 2.16. Let $\Lambda$ be an open subset of $\mathbb{R}^{d}$ and $\left\{f_{\mu}\right\}_{\mu \in \Lambda}$ be a continuous family of analytic functions on $[0,+\infty)$. Assume that, in a neighbourhood of some fixed $\hat{\mu} \in \Lambda$, there exist $n \geqslant 0$ continuous functions $\nu_{1}, \nu_{2}, \ldots, \nu_{n}$, with $\nu_{1}(\hat{\mu}), \nu_{2}(\hat{\mu}), \ldots, \nu_{n}(\hat{\mu})$ pairwise distinct, and such that the family $\left\{\mathscr{L}_{\boldsymbol{\nu}_{n}(\mu)}\left[f_{\mu}\right]\right\}_{\mu \in \Lambda}$ is continuously quantifiable in $\Lambda$ at $+\infty$ by $\xi(\mu)$. The following assertions hold:

(a) If $\xi(\hat{\mu})>-1$, then $\left\{\left(\mathscr{L}_{\boldsymbol{\nu}_{n}(\mu)} \circ \mathscr{F}\right)\left[f_{\mu}\right]\right\}_{\mu \in \Lambda}$ is continuously quantifiable in $\hat{\mu}$ at $+\infty$ by $\xi(\mu)$.

(b) If $\xi(\hat{\mu})<-1$, let us take $m \in \mathbb{N}$ such that $\xi(\hat{\mu})+2 m \in[-1,1)$. In this case:

(b1) If $M_{1}\left[\mathscr{L}_{\boldsymbol{\nu}_{n}(\mu)}\left[f_{\mu}\right]\right] \equiv M_{2}\left[\mathscr{L}_{\boldsymbol{\nu}_{n}(\mu)}\left[f_{\mu}\right]\right] \equiv \ldots \equiv M_{j-1}\left[\mathscr{L}_{\boldsymbol{\nu}_{n}(\mu)}\left[f_{\mu}\right]\right] \equiv 0$ and $M_{j}\left[\mathscr{L}_{\boldsymbol{\nu}_{n}(\hat{\mu})}\left[f_{\hat{\mu}}\right]\right] \neq 0$ for some $1 \leqslant j \leqslant m$, then $\left\{\left(\mathscr{L}_{\boldsymbol{\nu}_{n}(\mu)} \circ \mathscr{F}\right)\left[f_{\mu}\right]\right\}_{\mu \in \Lambda}$ is continuously quantifiable in $\hat{\mu}$ at $+\infty$ by $1-2 j$.

(b2) If $M_{1}\left[\mathscr{L}_{\boldsymbol{\nu}_{n}(\mu)}\left[f_{\mu}\right]\right] \equiv M_{2}\left[\mathscr{L}_{\boldsymbol{\nu}_{n}(\mu)}\left[f_{\mu}\right]\right] \equiv \ldots \equiv M_{m}\left[\mathscr{L}_{\boldsymbol{\nu}_{n}(\mu)}\left[f_{\mu}\right]\right] \equiv 0$ and $\xi(\hat{\mu})+2 m \notin\{-1,0\}$, then $\left\{\left(\mathscr{L}_{\boldsymbol{\nu}_{n}(\mu)} \circ \mathscr{F}\right)\left[f_{\mu}\right]\right\}_{\mu \in \Lambda}$ is continuously quantifiable in $\hat{\mu}$ at $+\infty$ by $\xi(\mu)$.

Proof. We first apply Proposition 2.14, which shows that $\mathscr{L}_{\boldsymbol{\nu}_{n}(\mu)}\left[f_{\mu}\right]$ is an analytic function on $[0,+\infty)$ for each $\mu \in \Lambda$, and that

$$
\left(\mathscr{L}_{\boldsymbol{\nu}_{n}(\mu)} \circ \mathscr{F}\right)\left[f_{\mu}\right](x)=\left(\mathscr{F} \circ \mathscr{L}_{\boldsymbol{\nu}_{n}(\mu)}\right)\left[f_{\mu}\right](x)=\int_{0}^{\frac{\pi}{2}} \mathscr{L}_{\boldsymbol{\nu}_{n}(\mu)}\left[f_{\mu}\right](x \sin \theta) d \theta
$$

Then the result follows by applying Theorem 2.10 to the family $\left\{\mathscr{L}_{\boldsymbol{\nu}_{n}(\mu)}\left[f_{\mu}\right]\right\}_{\mu \in \Lambda}$.

We point out that the assumption $M_{1}\left[\mathscr{L}_{\boldsymbol{\nu}_{n}(\mu)}\left[f_{\mu}\right]\right] \equiv M_{2}\left[\mathscr{L}_{\boldsymbol{\nu}_{n}(\mu)}\left[f_{\mu}\right]\right] \equiv \ldots \equiv M_{j-1}\left[\mathscr{L}_{\boldsymbol{\nu}_{n}(\mu)}\left[f_{\mu}\right]\right] \equiv 0$ in $(b 1)$ is void for $j=1$. Recall in addition that, by definition, $\mathscr{L}_{\boldsymbol{\nu}_{0}(\mu)}=i d$. Thus Proposition 2.16 with $n=0$ gives Theorem 2.10 as a particular case.

\section{Criticality of the period function at the outer boundary}

This section is devoted to prove the two main theoretical results of the paper. We consider analytic potential differential systems

$$
\left\{\begin{array}{l}
\dot{x}=-y \\
\dot{y}=V_{\mu}^{\prime}(x)
\end{array}\right.
$$


depending on a parameter $\mu \in \Lambda \subset \mathbb{R}^{d}$. Here, for each fixed $\mu \in \Lambda, V_{\mu}$ is an analytic function on a certain real interval $I_{\mu}$ that contains $x=0$. In what follows sometimes we shall also use the vector field notation $X_{\mu}:=-y \partial_{x}+V_{\mu}^{\prime}(x) \partial_{y}$ to refer to the above differential system. We suppose $V_{\mu}^{\prime}(0)=0$ and $V_{\mu}^{\prime \prime}(0)>0$, so that the origin is a non-degenerated center and we shall denote the projection of its period annulus $\mathscr{P}_{\mu}$ on the $x$-axis by $\mathcal{I}_{\mu}=\left(x_{\ell}(\mu), x_{r}(\mu)\right)$. Thus $x_{\ell}(\mu)<0<x_{r}(\mu)$. The corresponding Hamiltonian function is given by $H_{\mu}(x, y)=\frac{1}{2} y^{2}+V_{\mu}(x)$, where we fix that $V_{\mu}(0)=0$, and we set the energy level of the outer boundary of $\mathscr{P}_{\mu}$ to be $h_{0}(\mu)$, i.e. $H_{\mu}\left(\mathscr{P}_{\mu}\right)=\left(0, h_{0}(\mu)\right)$. Note then that $h_{0}(\mu)$ is a positive number or $+\infty$. In addition we define

$$
g_{\mu}(x):=x \sqrt{\frac{V_{\mu}(x)}{x^{2}}}=\operatorname{sgn}(x) \sqrt{V_{\mu}(x)}
$$

which is clearly a diffeomorphism from $\left(x_{\ell}(\mu), x_{r}(\mu)\right)$ to $\left(-\sqrt{h_{0}(\mu)}, \sqrt{h_{0}(\mu)}\right)$ due to $V_{\mu}(0)=V_{\mu}^{\prime}(0)=0$ and $V_{\mu}^{\prime \prime}(0)>0$. Recall in addition that, from $(2)$ and $(3)$, the period $T_{\mu}(h)$ of the periodic orbit of $X_{\mu}$ inside the energy level $\left\{H_{\mu}(x, y)=h\right\}$ verifies

$$
\sqrt{2} h^{2} T_{\mu}^{\prime}\left(h^{2}\right)=\mathscr{F}\left[f_{\mu}\right](h) \text {, with } f_{\mu}(x)=x\left(g_{\mu}^{-1}\right)^{\prime \prime}(x)-x\left(g_{\mu}^{-1}\right)^{\prime \prime}(-x),
$$

for all $h \in\left(0, \sqrt{h_{0}(\mu)}\right)$. Finally, it is also well known that $T_{\mu}$ is an analytic function on $\left(0, h_{0}(\mu)\right)$ which can be analytically extended to $h=0$.

Definition 3.1. Following the notation introduced just before, we say that the family of potential analytic differential systems $\left\{X_{\mu}\right\}_{\mu \in \Lambda}$ verifies the hypothesis $(\mathbf{H})$ in case that:

(a) For all $k \geqslant 0$, the map $(x, \mu) \longmapsto V_{\mu}^{(k)}(x)$ is continuous on $\left\{(x, \mu) \in \mathbb{R} \times \Lambda: x \in I_{\mu}\right\}$,

(b) $\mu \longmapsto x_{r}(\mu)$ is continuous on $\Lambda$ or $x_{r}(\mu)=+\infty$ for all $\mu \in \Lambda$,

(c) $\mu \longmapsto x_{\ell}(\mu)$ is continuous on $\Lambda$ or $x_{\ell}(\mu)=-\infty$ for all $\mu \in \Lambda$,

(d) $\mu \longmapsto h_{0}(\mu)$ is continuous on $\Lambda$ or $h_{0}(\mu)=+\infty$ for all $\mu \in \Lambda$.

Remark 3.2. Let $\left\{X_{\mu}\right\}_{\mu \in \Lambda}$ be a family of potential analytic differential systems verifying $(\mathbf{H})$. Then the outer boundary of its period annulus varies continuously in the sense of Definition 1.1. Indeed, to show this let $\gamma_{h, \mu}$ be the periodic orbit of $X_{\mu}$ inside the energy level $\left\{\frac{1}{2} y^{2}+V_{\mu}(x)=h\right\}$. Then

$$
d_{H}\left(\Pi_{\mu}, \Pi_{\hat{\mu}}\right) \leqslant d_{H}\left(\gamma_{h, \hat{\mu}}, \Pi_{\hat{\mu}}\right)+2 d_{H}\left(\gamma_{h, \hat{\mu}}, \gamma_{h, \mu}\right)+d_{H}\left(\gamma_{h, \mu}, \Pi_{\mu}\right),
$$

which tends to zero as $h \rightarrow h_{0}(\hat{\mu})$ and $\mu \rightarrow \hat{\mu}$ thanks to the hypothesis $(a)$ and $(d)$ in $(\mathbf{H})$.

Next two results are proved in [13].

Lemma 3.3. Let $\left\{X_{\mu}\right\}_{\mu \in \Lambda}$ be a family of potential analytic differential systems verifying $(\mathbf{H})$. Then the map $(z, \mu) \longmapsto g_{\mu}^{-1}(z)$ is continuous on the open $\operatorname{set}\left\{(z, \mu) \in \mathbb{R} \times \Lambda: z \in\left(-\sqrt{h_{0}(\mu)}, \sqrt{h_{0}(\mu)}\right)\right\}$.

Lemma 3.4. Let $\left\{X_{\mu}\right\}_{\mu \in \Lambda}$ be a family of potential analytic differential systems verifying $(\mathbf{H})$. Then

$$
\lim _{z \rightarrow-\sqrt{h_{0}(\mu)}} g_{\mu}^{-1}(z)=x_{\ell}(\mu) \text { and } \lim _{z \rightarrow \sqrt{h_{0}(\mu)}} g_{\mu}^{-1}(z)=x_{r}(\mu)
$$

uniformly on every compact subset of $\Lambda$. Moreover, if $h_{0}, x_{\ell}$ and $x_{r}$ are finite at $\mu=\hat{\mu}$, then $(z, \mu) \longmapsto g_{\mu}^{-1}(z)$ extends continuously to $\left(-\sqrt{h_{0}(\hat{\mu})}, \hat{\mu}\right)$ and $\left(\sqrt{h_{0}(\hat{\mu})}, \hat{\mu}\right)$ for all $\mu \in \Lambda$.

Next two sections are concerned with the criticality at the outer boundary of potential systems verifying the hypothesis $(\mathbf{H})$. Section 3.1 is devoted to prove Theorem A, that deals with the case $h_{0} \equiv+\infty$, whereas in Section 3.2 we prove Theorem B, that tackle the case in which $h_{0}$ is finite. 


\subsection{Potential systems with infinite energy}

In this section we shall study the criticality at the outer boundary of the period annulus for families of potential systems such that $h_{0}(\mu)=+\infty$ for all $\mu \in \Lambda$. The idea is to take a non-vanishing function $f$ and find sufficient conditions in order that $f T_{\mu}^{\prime}$ can be embedded into the simplest ECT-system we can consider, namely $\left(h^{\nu_{1}(\mu)}, h^{\nu_{2}(\mu)}, \ldots, h^{\nu_{n}(\mu)}\right)$. We precise this in the following result.

Lemma 3.5. Let $\left\{X_{\mu}\right\}_{\mu \in \Lambda}$ be a family of potential analytic differential systems verifying $(\mathbf{H})$ and such that $h_{0} \equiv+\infty$. Assume that there exist $n \geqslant 1$ continuous functions $\nu_{1}, \nu_{2} \ldots, \nu_{n}$ in a neighbourhood of some fixed $\hat{\mu} \in \Lambda$ and an analytic non-vanishing function $f$ on $(0,+\infty)$ such that

$$
\lim _{h \rightarrow+\infty} h^{\nu_{n}(\mu)} W\left[h^{\nu_{1}(\mu)}, \ldots, h^{\nu_{n-1}(\mu)}, f(h) T_{\mu}^{\prime}(h)\right]=\Delta(\mu),
$$

uniformly in $\mu \approx \hat{\mu}$, and $\Delta(\hat{\mu}) \neq 0$. Then $\operatorname{Crit}\left(\left(\Pi_{\hat{\mu}}, X_{\hat{\mu}}\right), X_{\mu}\right) \leqslant n-1$.

Proof. Note first that $\nu_{1}, \nu_{2} \ldots, \nu_{n-1}$ must be pairwise distinct at $\mu=\hat{\mu}$ because $\Delta(\hat{\mu}) \neq 0$. Thus, by continuity, for each $k=1,2, \ldots, n-1$ we have that $W\left[h^{\nu_{1}(\mu)}, \ldots, h^{\nu_{k}(\mu)}\right] \neq 0$ for all $h>0$ and $\mu \approx \hat{\mu}$. On the other hand, by the uniformity of the limit as $h$ tends to $+\infty$ and the assumption $\Delta(\hat{\mu}) \neq 0$, there exist $M>0$ and a neighbourhood $U$ of $\hat{\mu}$ such that

$$
W\left[h^{\nu_{1}(\mu)}, \ldots, h^{\nu_{n-1}(\mu)}, f(h) T_{\mu}^{\prime}(h)\right] \neq 0 \text { for } h \in(M,+\infty) \text { and } \mu \in U .
$$

Then, by Lemma 2.3 we can assert that $\left(h^{\nu_{1}(\mu)}, \ldots, h^{\nu_{n-1}(\mu)}, f(h) T_{\mu}^{\prime}(h)\right)$ is an ECT-system on $(M,+\infty)$ for all $\mu \in U$. In particular, since $f$ is a unity, $T_{\mu}^{\prime}$ has no more than $n-1$ isolated zeros on $(M,+\infty)$ for $\mu \approx \hat{\mu}$, counted with multiplicities. We claim that this implies $\operatorname{Crit}\left(\left(\Pi_{\hat{\mu}}, X_{\hat{\mu}}\right), X_{\mu}\right) \leqslant n-1$, see Definition 1.1. To show this notice first that, by Remark 3.2, the outer boundary of the period annulus varies continuously. Suppose, by contradiction, that there exist $n$ sequences $\left\{\gamma_{\mu_{i}}^{k}\right\}_{i \in \mathbb{N}}, k=1,2, \ldots, n$, where each $\gamma_{\mu_{i}}^{k}$ is a critical periodic orbit of $X_{\mu_{i}}$, such that $\mu_{i} \rightarrow \hat{\mu}$ and $d_{H}\left(\gamma_{\mu_{i}}^{k}, \Pi_{\hat{\mu}}\right) \rightarrow 0$ as $i \rightarrow+\infty$. Then, due to

$$
d_{H}\left(\gamma_{\mu_{i}}^{k}, \Pi_{\mu_{i}}\right) \leqslant d_{H}\left(\gamma_{\mu_{i}}^{k}, \Pi_{\hat{\mu}}\right)+d_{H}\left(\Pi_{\mu_{i}}, \Pi_{\hat{\mu}}\right)
$$

we have that $d_{H}\left(\gamma_{\mu_{i}}^{k}, \Pi_{\mu_{i}}\right)$ tends to zero as $i \rightarrow+\infty$. This contradicts that, for all $\mu \in U, T_{\mu}^{\prime}$ has no more than $n-1$ isolated zeros on $(M,+\infty)$. So the claim is true and the result follows.

The proof of the following result is a straightforward application of [13, Lemma 3.5]. For the sake of brevity we omit it here but we refer the reader to the proof of Lemma 3.11, which follows similarly.

Lemma 3.6. Let $\left\{X_{\mu}\right\}_{\mu \in \Lambda}$ be a family of potential analytic systems verifying $(\mathbf{H})$ and such that $h_{0} \equiv+\infty$. Let $\left\{f_{\mu}\right\}_{\mu \in \Lambda}$ be a continuous family of analytic functions which is continuously quantifiable in $\Lambda$ at $x=x_{r}(\mu)$ (respectively, $\left.x=x_{\ell}(\mu)\right)$ by $\alpha(\mu)$ with limit $a(\mu)$. Assume moreover that $\left\{V_{\mu}\right\}_{\mu \in \Lambda}$ is continuously quantifiable in $\Lambda$ at $x=x_{r}(\mu)$ (respectively, $x=x_{\ell}(\mu)$ ) by $\beta(\mu)$ with limit $b(\mu)$. Then, $\left\{f_{\mu} \circ g_{\mu}^{-1}\right\}_{\mu \in \Lambda}$ is continuously quantifiable at $+\infty$ (respectively, $-\infty)$ by $2(\alpha / \beta)(\mu)$ with limit $\left(a b^{-\alpha / \beta}\right)(\mu)$.

We can now state our result concerning the criticality at the outer boundary for the case $h_{0} \equiv+\infty$. In its statement, and from now on, for a given function $f:(-a, a) \longrightarrow \mathbb{R}$, we denote $\mathcal{P}[f](x):=f(x)+f(-x)$. Let us also remark that the assumption requiring the existence of functions $\nu_{1}, \nu_{2}, \ldots, \nu_{n}$ is void in case that $n=0$.

Theorem A. Let $\left\{X_{\mu}\right\}_{\mu \in \Lambda}$ be a family of potential analytic systems verifying $(\mathbf{H})$ and such that $h_{0} \equiv+\infty$. Assume that there exist $n \geqslant 0$ continuous functions $\nu_{1}, \nu_{2}, \ldots, \nu_{n}$ in a neighbourhood of some fixed $\hat{\mu} \in \Lambda$ such that the family $\left\{\left(\mathscr{L}_{\boldsymbol{\nu}_{n}(\mu)} \circ \mathcal{P}\right)\left[z\left(g_{\mu}^{-1}\right)^{\prime \prime}(z)\right]\right\}_{\mu \in \Lambda}$ is continuously quantifiable in $\Lambda$ at $+\infty$ by $\xi(\mu)$. For each $i \in \mathbb{N}$, let $M_{i}(\mu)$ be the $i$-th momentum of $\left(\mathscr{L}_{\boldsymbol{\nu}_{n}(\mu)} \circ \mathcal{P}\right)\left[z\left(g_{\mu}^{-1}\right)^{\prime \prime}(z)\right]$, whenever it is well defined. The following assertions hold:

(a) If $\xi(\hat{\mu})>-1$, then $\operatorname{Crit}\left(\left(\Pi_{\hat{\mu}}, X_{\hat{\mu}}\right), X_{\mu}\right) \leqslant n$. 
(b) If $\xi(\hat{\mu})<-1$, let $m \in \mathbb{N}$ be such that $\xi(\hat{\mu})+2 m \in[-1,1)$. Then $\operatorname{Crit}\left(\left(\Pi_{\hat{\mu}}, X_{\hat{\mu}}\right), X_{\mu}\right) \leqslant n$ in case that

(b1) either $M_{1} \equiv M_{2} \equiv \ldots \equiv M_{j-1} \equiv 0$ and $M_{j}(\hat{\mu}) \neq 0$ for some $j \in\{1,2, \ldots, m\}$,

(b2) or $M_{1} \equiv M_{2} \equiv \ldots \equiv M_{m} \equiv 0$ and $\xi(\hat{\mu})+2 m \notin\{-1,0\}$.

Finally, if the following conditions are verified, then $\left\{\left(\mathscr{L}_{\boldsymbol{\nu}_{n}(\mu)} \circ \mathcal{P}\right)\left[z\left(g_{\mu}^{-1}\right)^{\prime \prime}(z)\right]\right\}_{\mu \in \Lambda}$ is continuously quantifiable at $+\infty$ by $\xi(\mu)=2 \max \left\{\left(\frac{\alpha_{\ell}}{\beta_{\ell}}\right)(\mu),\left(\frac{\alpha_{r}}{\beta_{r}}\right)(\mu)\right\}+(n+1)^{2}-\sum_{i=1}^{n} \nu_{i}(\mu)$ :

(i) $\left\{V_{\mu}\right\}_{\mu \in \Lambda}$ is continuously quantifiable at $x_{\ell}(\mu)$ by $\beta_{\ell}(\mu)$ and at $x_{r}(\mu)$ by $\beta_{r}(\mu)$ with limits $b_{\ell}(\mu)$ and $b_{r}(\mu)$, respectively,

(ii) setting $\mathscr{R}_{\mu}:=\frac{\left(V_{\mu}^{\prime}\right)^{2}-2 V_{\mu} V_{\mu}^{\prime \prime}}{\left(V_{\mu}^{\prime}\right)^{3}}$, the function $x \longmapsto V_{\mu}^{\prime}(x)^{-\frac{n(n+1)}{2}} W\left[V_{\mu}^{\frac{\nu_{1}(\mu)-1}{2}}, \ldots, V_{\mu}^{\frac{\nu_{n}(\mu)-1}{2}}, \mathscr{R}_{\mu}\right](x)$ is continuously quantifiable at $x_{\ell}(\mu)$ by $\alpha_{\ell}(\mu)$ and at $x_{r}(\mu)$ by $\alpha_{r}(\mu)$ with limits $a_{\ell}(\mu)$ and $a_{r}(\mu)$, respectively,

(iii) and either $\frac{\alpha_{\ell}}{\beta_{\ell}}(\mu) \neq \frac{\alpha_{r}}{\beta_{r}}(\mu)$ or, otherwise, $\left(a_{\ell}\left(b_{r}\right)^{\frac{\alpha_{r}}{\beta_{r}}}-(-1)^{\frac{n(n+1)}{2}} a_{r}\left(b_{\ell}\right)^{\frac{\alpha_{\ell}}{\beta_{\ell}}}\right)(\mu) \neq 0$.

Proof. Denote $f_{\mu}(z):=\mathcal{P}\left[z\left(g_{\mu}^{-1}\right)^{\prime \prime}(z)\right]$ for shortness. Then Lemma 3.3 and the hypothesis $(\mathbf{H})$ guarantee that $\left\{f_{\mu}\right\}_{\mu \in \Lambda}$ is a continuous family of analytic functions on $[0,+\infty)$. From $(7), \sqrt{2} h^{2} T_{\mu}^{\prime}\left(h^{2}\right)=\mathscr{F}\left[f_{\mu}\right](h)$ for all $h \in(0,+\infty)$, and therefore it suffices to prove that there exist $M, \varepsilon>0$ such that $\mathscr{F}\left[f_{\mu}\right](h)$ has at most $n$ isolated zeroes counted with multiplicities for $h>M$ and $\|\mu-\hat{\mu}\|<\varepsilon$.

Since $\xi(\mu)$ is the quantifier of $\left\{\mathscr{L}_{\boldsymbol{\nu}_{n}(\mu)}\left[f_{\mu}\right]\right\}_{\mu \in \Lambda}$ at $+\infty$, by applying Proposition 2.16 we can assert that $\left\{\left(\mathscr{L}_{\boldsymbol{\nu}_{n}(\mu)} \circ \mathscr{F}\right)\left[f_{\mu}\right]\right\}_{\mu \in \Lambda}$ is continuously quantifiable in $\hat{\mu}$ at $+\infty$ by $\nu_{n+1}(\mu):=\xi(\mu)$, in cases $(a)$ and $(b 2)$, and by $\nu_{n+1}(\mu):=1-2 j$, in case $(b 1)$. Then, taking account of the definition of $\mathscr{L}_{\boldsymbol{\nu}_{n}(\mu)}$, see $(4)$, in these cases we get that

$$
\lim _{(h, \mu) \rightarrow(+\infty, \hat{\mu})} h^{-\nu_{n+1}(\mu)} \frac{W\left[h^{\nu_{1}(\mu)}, \ldots, h^{\nu_{n}(\mu)}, \mathscr{F}\left[f_{\mu}\right](h)\right]}{h^{\sum_{i=1}^{n}\left(\nu_{i}(\mu)-i\right)}} \neq 0 .
$$

Thus, since $\mathscr{F}\left[f_{\mu}\right](h)=\sqrt{2} h^{2} T_{\mu}^{\prime}\left(h^{2}\right)$, by applying Lemma 3.5 we have that $\operatorname{Crit}\left(\left(\Pi_{\hat{\mu}}, X_{\hat{\mu}}\right), X_{\mu}\right) \leqslant n$, as desired. This proves the first part of the result.

Let us turn now to the proof of second part of the result. With this aim in view we note that if $\phi$ is any analytic function on $(-a, a)$, then

$$
\mathscr{L}_{\boldsymbol{\nu}_{n}}[\mathcal{P} \circ \phi](x)=\frac{W\left[x^{\nu_{1}}, x^{\nu_{2}}, \ldots, x^{\nu_{n}}, \phi(x)\right]}{x^{\sum_{i=1}^{n}\left(\nu_{i}-i\right)}}+\frac{W\left[x^{\nu_{1}}, x^{\nu_{2}}, \ldots, x^{\nu_{n}}, \phi(-x)\right]}{x^{\sum_{i=1}^{n}\left(\nu_{i}-i\right)}} \text { for all } x \in(0, a) .
$$

Let us set $\Delta(\mu):=\sum_{i=1}^{n}\left(\nu_{i}(\mu)-i\right)$ for shortness. Since one can verify that $\left(g^{-1}(z)\right)^{\prime \prime}=2 \mathscr{R}\left(g^{-1}(z)\right)$ with $\mathscr{R}=\frac{\left(V^{\prime}\right)^{2}-2 V V^{\prime \prime}}{\left(V^{\prime}\right)^{3}}$ and $V\left(g^{-1}(z)\right)=z^{2}$, by applying Lemma 2.11 some computations show that

$$
\frac{W\left[z^{\nu_{1}(\mu)}, z^{\nu_{2}(\mu)}, \ldots, z^{\nu_{n}(\mu)}, z\left(g_{\mu}^{-1}\right)^{\prime \prime}(z)\right]}{z^{\Delta(\mu)}}=2^{1+\frac{n(n+1)}{2}} S_{\mu}\left(g_{\mu}^{-1}(z)\right) \text {, for all } z>0,
$$

where

$$
S_{\mu}(x):=\frac{W\left[V_{\mu}^{\frac{\nu_{1}(\mu)-1}{2}}, \ldots, V_{\mu}^{\frac{\nu_{n}(\mu)-1}{2}}, \mathscr{R}_{\mu}\right](x)}{V_{\mu}^{\prime}(x)^{\frac{n(n+1)}{2}} V_{\mu}(x)^{\frac{\Delta(\mu)}{2}-\frac{(n+1)(n+2)}{4}}} .
$$

Similarly, due to $V\left(g^{-1}(-z)\right)=z^{2}$,

$$
\frac{W\left[z^{\nu_{1}}, z^{\nu_{2}}, \ldots, z^{\nu_{n}},-z\left(g_{\mu}^{-1}\right)^{\prime \prime}(-z)\right]}{z^{\Delta(\mu)}}=(-2)^{1+\frac{n(n+1)}{2}} S_{\mu}\left(g_{\mu}^{-1}(-z)\right) \text {, for all } z>0 .
$$

Accordingly, taking (8) with $\phi(z)=z\left(g_{\mu}^{-1}\right)^{\prime \prime}(z)$, it turns out that the quantifiers of $S_{\mu} \circ g_{\mu}^{-1}$ at $+\infty$ and at $-\infty$ will "generically" determine the quantifier of $\mathscr{L}_{\boldsymbol{\nu}_{n}(\mu)}\left[f_{\mu}\right]$ at $+\infty$. 
Henceforth, for the sake of shortness, we omit the unessential dependence with respect to $\mu$. On account of $(i)$ and $(i i)$ it follows that $\left\{S_{\mu}\right\}_{\mu \in \Lambda}$ is continuously quantifiable at $x_{\ell}$ by $\alpha_{\ell}-\beta_{\ell}\left(\frac{\Delta}{2}-\frac{(n+1)(n+2)}{4}\right)$ and at $x_{r}$ by $\alpha_{r}-\beta_{r}\left(\frac{\Delta}{2}-\frac{(n+1)(n+2)}{4}\right)$, with limits $a_{\ell} b_{\ell}^{\frac{(n+1)(n+2)}{4}-\frac{\Delta}{2}}$ and $a_{r} b_{r}^{\frac{(n+1)(n+2)}{4}}-\frac{\Delta}{2}$, respectively. Then, by applying Lemma 3.6 and using $(i)$ again, $\left\{S_{\mu} \circ g_{\mu}^{-1}\right\}_{\mu \in \Lambda}$ is continuously quantifiable in $\Lambda$ at $-\infty$ by $2 \frac{\alpha_{\ell}}{\beta_{\ell}}-\Delta+$ $\frac{(n+1)(n+2)}{2}$ and at $+\infty$ by $2 \frac{\alpha_{r}}{\beta_{r}}-\Delta+\frac{(n+1)(n+2)}{2}$, with limits $a_{\ell}\left(b_{\ell}\right)^{-\frac{\alpha_{\ell}}{\beta_{\ell}}}$ and $a_{r}\left(b_{r}\right)^{-\frac{\alpha_{r}}{\beta_{r}}}$, respectively. Finally, again from (8) with $\phi(z)=z\left(g_{\mu}^{-1}\right)^{\prime \prime}(z)$, the assumption $(i i i)$ gurarantees that $\left\{\left(\mathscr{L}_{\boldsymbol{\nu}_{n}(\mu)} \circ \mathcal{P}\right)\left[z\left(g_{\mu}^{-1}\right)^{\prime \prime}(z)\right]\right\}_{\mu \in \Lambda}$ is continuously quantifiable at $+\infty$ by $\xi=2 \max \left\{\left(\frac{\alpha_{\ell}}{\beta_{\ell}}\right),\left(\frac{\alpha_{r}}{\beta_{r}}\right)\right\}-\Delta+\frac{(n+1)(n+2)}{2}$. This completes the proof of the result because one can easily verify that $\frac{(n+1)(n+2)}{2}-\Delta=(n+1)^{2}-\sum_{i=1}^{n} \nu_{i}$.

\subsection{Potential systems with finite energy}

In this section we shall study the criticality at the outer boundary of the period annulus for families of potential systems with $h_{0}(\mu)<+\infty$ for all $\mu \in \Lambda$. If we proceed the same way as for the case $h_{0}=+\infty$, we would take an appropriate non-vanishing function $f$ and try to embed $f T_{\mu}^{\prime}$ into some easy ECT-system. To this end the natural candidate is $\left(\left(h_{0}(\mu)-h\right)^{\nu_{1}(\mu)},\left(h_{0}(\mu)-h\right)^{\nu_{2}(\mu)}, \ldots,\left(h_{0}(\mu)-h\right)^{\nu_{n}(\mu)}\right)$. However we did not succeed with such an approach. Instead we shall take advantage of Proposition 2.16, which is in fact addressed to the case $h_{0}=+\infty$. This forces us to "translate" the case $h_{0}<+\infty$ to the case $h_{0}=+\infty$ and gives rise to some technicalities that make things more complicated than it should be. With this aim in view we define next a differential operator which is conjugated to $\mathscr{L}_{\boldsymbol{\nu}_{n}}$. The conjugation is precisely the tool that enables us to translate the case $h_{0}<+\infty$ to the case $h_{0}=+\infty$ and apply Proposition 2.16.

Given $\nu_{1}, \ldots, \nu_{n} \in \mathbb{R}$, in this section we consider the linear ordinary differential operator

$$
\mathscr{D}_{\nu_{n}}: \mathscr{C}^{\omega}((0,1)) \longrightarrow \mathscr{C}^{\omega}((0,1))
$$

defined by

$$
\mathscr{D}_{\nu_{n}}[f](x):=\left(x\left(1-x^{2}\right)\right)^{\frac{n(n+1)}{2}} \frac{W\left[\psi_{\nu_{1}}, \ldots, \psi_{\nu_{n}}, f\right](x)}{\prod_{i=1}^{n} \psi_{\nu_{i}}(x)},
$$

where as usual we use the notation $\boldsymbol{\nu}_{n}=\left(\nu_{1}, \ldots, \nu_{n}\right)$ and

$$
\psi_{\nu}(x):=\frac{1}{1-x^{2}}\left(\frac{x}{\sqrt{1-x^{2}}}\right)^{\nu} .
$$

In addition we define $\mathscr{D}_{\boldsymbol{\nu}_{0}}:=i d$ for the sake of convenience. Setting

$$
\phi(x):=\frac{x}{\sqrt{1+x^{2}}},
$$

we also consider the operator $\mathscr{B}: \mathscr{C}^{\omega}([0,1)) \longrightarrow \mathscr{C}^{\omega}([0,+\infty))$ defined by

$$
\mathscr{B}[f](x):=\left(1-\phi^{2}(x)\right)(f \circ \phi)(x)=\frac{1}{1+x^{2}}(f \circ \phi)(x) .
$$

We will show next that $\mathscr{B}$ conjugates $\mathscr{D}_{\boldsymbol{\nu}_{n}}$ and $\mathscr{L}_{\boldsymbol{\nu}_{n}}$. This fact eventually will enable us to take advantage of Proposition 2.16. Before proving it we introduce the following definition.

Definition 3.7. Let $f$ be an analytic function on $[0,1)$. Then, for each $n \in \mathbb{N}$ we call

$$
N_{n}[f]:=\int_{0}^{1} \frac{f(x)}{\sqrt{1-x^{2}}}\left(\frac{x}{\sqrt{1-x^{2}}}\right)^{2 n-2} d x
$$

the $n$-th momentum of $f$, whenever it is well defined.

Lemma 3.8. Consider $\nu_{1}, \nu_{2}, \ldots, \nu_{n} \in \mathbb{R}$. Then the following hold: 
(a) $\mathscr{B}\left[\psi_{\nu_{i}}\right](x)=x^{\nu_{i}}$ for $i=1,2, \ldots, n$.

(b) $\mathscr{B} \circ \mathscr{D}_{\boldsymbol{\nu}_{n}}=\mathscr{L}_{\boldsymbol{\nu}_{n}} \circ \mathscr{B}$.

(c) $(\mathscr{F} \circ \mathscr{B})[f](x)=\sqrt{1+x^{2}}(\mathscr{B} \circ \mathscr{F})[f](x)$ for any $f \in \mathscr{C}^{\omega}((0,1))$.

(d) $N_{n}=M_{n} \circ \mathscr{B}$.

Proof. Let us show $(b)$ because $(a)$ follows straightforward. So take $f \in \mathscr{C}^{\omega}((0,1))$ and note that

$$
\left(\mathscr{B} \circ \mathscr{D}_{\nu_{n}}\right)[f](x)=\left(1-\phi(x)^{2}\right)\left(\phi(x)\left(1-\phi(x)^{2}\right)\right)^{\frac{n(n+1)}{2}} \frac{W\left[\psi_{\nu_{1}}, \ldots, \psi_{\nu_{n}}, f\right](\phi(x))}{\prod_{i=1}^{n} \psi_{\nu_{i}}(\phi(x))}
$$

by definition. On the other hand, by applying Lemma 2.11 we get

$$
\begin{aligned}
W\left[\psi_{\nu_{1}}, \ldots, \psi_{\nu_{n}}, f\right](\phi(x)) & =\left(1-\phi(x)^{2}\right)^{-n-1}\left(\phi^{\prime}(x)\right)^{-\frac{n(n+1)}{2}} W\left[\mathscr{B}\left[\psi_{\nu_{1}}\right], \ldots, \mathscr{B}\left[\psi_{\nu_{n}}\right], \mathscr{B}[f]\right](x) \\
& =\left(1-\phi(x)^{2}\right)^{-n-1} x^{\frac{n(n+1)}{2}}\left(\phi(x)\left(1-\phi(x)^{2}\right)\right)^{-\frac{n(n+1)}{2}} W\left[x^{\nu_{1}}, \ldots, x^{\nu_{n}}, \mathscr{B}[f](x)\right],
\end{aligned}
$$

where in the second equality we use $(a)$ and that $x \phi^{\prime}(x)=\phi(x)\left(1-\phi(x)^{2}\right)$. Consequently, since on account of $(a)$ we have $\psi_{\nu}(\phi(x))=\frac{x^{\nu}}{1-\phi(x)^{2}}$, the combination of the two previous indented equalities gives

$$
\left(\mathscr{B} \circ \mathscr{D}_{\boldsymbol{\nu}_{n}}\right)[f](x)=\frac{W\left[x^{\nu_{1}}, \ldots, x^{\nu_{n}}, \mathscr{B}[f](x)\right]}{x^{\sum_{i=1}^{n}\left(\nu_{i}-i\right)}}=\left(\mathscr{L}_{\boldsymbol{\nu}_{n}} \circ \mathscr{B}\right)[f](x),
$$

as desired. Let us turn now to the proof of $(c)$. Take any $s \in(0,1)$ and $h \in \mathscr{C}^{\omega}([0,1))$ and note that the change of variable $u=s \sin \theta$ gives

$$
\mathscr{F}[h](s)=\int_{0}^{\frac{\pi}{2}} h(s \sin \theta) d \theta=\int_{0}^{s} \frac{h(u)}{\sqrt{s^{2}-u^{2}}} d u .
$$

If $f$ is any analytic function on $[0,1)$, then performing the change of variable $z=\phi(x)$ it follows that

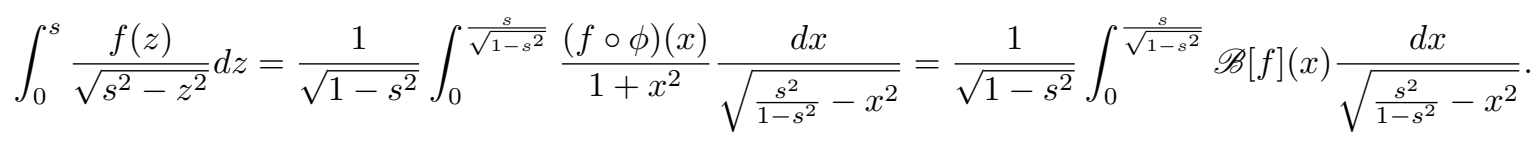

Accordingly, by applying above the equality in (11) with $h=f$ and $h=\mathscr{B}[f]$, we get

$$
\mathscr{F}[f](s)=\frac{1}{\sqrt{1-s^{2}}}(\mathscr{F} \circ \mathscr{B})[f]\left(\frac{s}{\sqrt{1-s^{2}}}\right) .
$$

Finally the composition with $\mathscr{B}$ on both sides of this equality and an easy computation yields to

$$
\begin{aligned}
(\mathscr{B} \circ \mathscr{F})[f](s) & =\left(1-\phi(s)^{2}\right) \frac{1}{\sqrt{1-\phi(s)^{2}}}(\mathscr{F} \circ \mathscr{B})[f]\left(\frac{\phi(s)}{\sqrt{1-\phi(s)^{2}}}\right) \\
& =\left(1+s^{2}\right)^{-\frac{1}{2}}(\mathscr{F} \circ \mathscr{B})[f](s),
\end{aligned}
$$

which shows $(c)$. Finally let us prove $(d)$. If $f$ is an analytic function on $[0,1)$, then by means of the change of variable $z=\phi(x)$ once again we get

$$
N_{n}[f]=\int_{0}^{1} \frac{f(z)}{\sqrt{1-z^{2}}}\left(\frac{z}{\sqrt{1-z^{2}}}\right)^{2 n-2} d z=\int_{0}^{+\infty} \frac{(f \circ \phi)(x)}{1+x^{2}} x^{2 n-2} d x=M_{n}[\mathscr{B}[f]]
$$

as desired. This completes the proof of the result.

Lemma 3.9. Let $\left\{f_{\mu}\right\}_{\mu \in \Lambda}$ be a continuous family of analytic functions on $[0,1)$. Then $\left\{f_{\mu}\right\}_{\mu \in \Lambda}$ is continuously quantifiable in $\Lambda$ at $z=1$ by $\alpha(\mu)$ if and only if $\left\{\mathscr{B}\left[f_{\mu}\right]\right\}_{\mu \in \Lambda}$ is continuously quantifiable in $\Lambda$ at $+\infty$ by $2 \alpha(\mu)-2$. 
Proof. By definition, $\mathscr{B}\left[f_{\mu}\right](x)=\frac{1}{1+x^{2}} f_{\mu}(\phi(x))$ with $\phi(x)=\frac{x}{\sqrt{1+x^{2}}}$. Therefore, for a given $\hat{\mu} \in \Lambda$,

$$
\begin{aligned}
\lim _{(x, \mu) \rightarrow(+\infty, \hat{\mu})} \frac{\mathscr{B}\left[f_{\mu}\right](x)}{x^{2 \alpha(\mu)-2}} & =\lim _{(x, \mu) \rightarrow(+\infty, \hat{\mu})} \frac{f_{\mu}(\phi(x))}{\left(1+x^{2}\right) x^{2 \alpha(\mu)-2}}=\lim _{(x, \mu) \rightarrow(+\infty, \hat{\mu})} \frac{f_{\mu}(\phi(x))}{\left(1+x^{2}\right)^{\alpha(\mu)}} \\
& =\lim _{(z, \mu) \rightarrow(1, \hat{\mu})} \frac{f_{\mu}(z)}{\left(1+\phi^{-1}(z)^{2}\right)^{\alpha(\mu)}}=\lim _{(z, \mu) \rightarrow(1, \hat{\mu})} f_{\mu}(z)\left(1-z^{2}\right)^{\alpha(\mu)}
\end{aligned}
$$

where we used that $\phi^{-1}(z)=\frac{z}{\sqrt{1-z^{2}}}$. Since the first limit is different from zero if and only if the last one is different from zero, the result follows.

We shall bound the criticality at the outer boundary by means of the following result.

Lemma 3.10. Let $\left\{X_{\mu}\right\}_{\mu \in \Lambda}$ be a family of potential analytic differential systems verifying $(\mathbf{H})$ and such that $\mu \longmapsto h_{0}(\mu)$ is continuous on $\Lambda$. Assume that there exist $n \geqslant 1$ continuous functions $\nu_{1}, \nu_{2} \ldots, \nu_{n}$ in a neighbourhood of some fixed $\hat{\mu} \in \Lambda$ and an analytic non-vanishing function $f$ on $(0,1)$ such that

$$
\lim _{z \longrightarrow 1}(1-z)^{\nu_{n}(\mu)} W\left[\psi_{\nu_{1}(\mu)}(z), \ldots, \psi_{\nu_{n-1}(\mu)}(z), f(z) T_{\mu}^{\prime}\left(z^{2} h_{0}(\mu)\right)\right]=\Delta(\mu),
$$

uniformly in $\mu \approx \hat{\mu}$, and $\Delta(\hat{\mu}) \neq 0$. Then $\operatorname{Crit}\left(\left(\Pi_{\hat{\mu}}, X_{\hat{\mu}}\right), X_{\mu}\right) \leqslant n-1$.

Proof. Note first that $\nu_{1}, \nu_{2} \ldots, \nu_{n-1}$ must be pairwise distinct at $\mu=\hat{\mu}$ because $\Delta(\hat{\mu}) \neq 0$. Consequently, since $\mathscr{B}\left[\psi_{\nu_{i}(\mu)}\right](x)=x^{\nu_{i}(\mu)}$ due to $(a)$ in Lemma 3.8, by applying Lemmas 2.11 and 2.13 we can assert that $W\left[\psi_{\nu_{1}(\mu)}, \ldots, \psi_{\nu_{k}(\mu)}\right](z) \neq 0$ for all $z \in(0,1)$ and $\mu \approx \hat{\mu}, k=1,2, \ldots, n-1$. On the other hand, by the uniformity of the limit as $z \longrightarrow 1$ and the hypothesis $\Delta(\hat{\mu}) \neq 0$, there exist $\varepsilon>0$ and a neighbourhood $U$ of $\hat{\mu}$ such that

$$
W\left[\psi_{\nu_{1}(\mu)}(z), \ldots, \psi_{\nu_{n-1}(\mu)}(z), f(z) T_{\mu}^{\prime}\left(z^{2} h_{0}(\mu)\right)\right] \neq 0 \text { for all } z \in(1-\varepsilon, 1) \text { and } \mu \in U .
$$

Accordingly, by Lemma 2.3, $\left(\psi_{\nu_{1}(\mu)}(z), \ldots, \psi_{\nu_{n-1}(\mu)}(z), f(z) T_{\mu}^{\prime}\left(z^{2} h_{0}(\mu)\right)\right)$ is an ECT-system on $(1-\varepsilon, 1)$ for all $\mu \in U$. In particular, since $f$ is a unity, there exists $\delta>0$ such that $T_{\mu}^{\prime}(h)$ has no more than $n-1$ zeros on $\left(h_{0}(\mu)-\delta, h_{0}(\mu)\right)$, counted with multiplicities, for all $\mu \in U$. From here the proof follows verbatim what we do to show Lemma 3.5 and so for the sake of brevity we omit it.

Lemma 3.11. Let $\left\{X_{\mu}\right\}_{\mu \in \Lambda}$ be a family of potential analytic systems verifying $(\mathbf{H})$ such that $h_{0}(\mu)<+\infty$ for all $\mu \in \Lambda$. Let $\left\{f_{\mu}\right\}_{\mu \in \Lambda}$ be a continuous family of analytic functions which is continuously quantifiable in $\Lambda$ at $x=x_{r}(\mu)$ (respectively, $\left.x=x_{\ell}(\mu)\right)$ by $\alpha(\mu)$ with limit $a(\mu)$. Assume moreover that $\left\{h_{0}(\mu)-V_{\mu}\right\}_{\mu \in \Lambda}$ is continuously quantifiable in $\Lambda$ at $x=x_{r}(\mu)$ (respectively, $x=x_{\ell}(\mu)$ ) by $\beta(\mu)$ with limit $b(\mu)$. Then, $\left\{\left(f_{\mu} \circ g_{\mu}^{-1}\right)\left(z \sqrt{h_{0}(\mu)}\right)\right\}_{\mu \in \Lambda}$ is continuously quantifiable at $z=1$ (respectively, $z=-1$ ) by $-(\alpha / \beta)(\mu)$ with limit $\left(a\left(2 h_{0} / b\right)^{\alpha / \beta}\right)(\mu)$.

Proof. We show the result for $z=1$ (the case $z=-1$ follows exactly the same way). By Lemma 3.4, we know that $g_{\mu}^{-1}\left(z \sqrt{h_{0}(\mu)}\right)$ tends to $x_{r}(\mu)$ uniformly on $\mu$ as $z \longrightarrow 1$. Therefore, since $g_{\mu}^{2}=V_{\mu}$,

$$
\lim _{z \rightarrow 1} \frac{\left(f_{\mu} \circ g_{\mu}^{-1}\right)\left(z \sqrt{h_{0}(\mu)}\right)}{\left(1-z^{2}\right)^{\left(\frac{\alpha}{\beta}\right)(\mu)}}=\lim _{x \rightarrow x_{r}(\mu)} \frac{h_{0}(\mu)^{\left(\frac{\alpha}{\beta}\right)(\mu)} f_{\mu}(x)}{\left(h_{0}(\mu)-V_{\mu}(x)\right)^{\left(\frac{\alpha}{\beta}\right)(\mu)}}=\left(a\left(h_{0} / b\right)^{\alpha / \beta}\right)(\mu)
$$

uniformly on $\mu$. Taking any $\hat{\mu} \in \Lambda$, this shows that

$$
\lim _{(z, \mu) \rightarrow(1, \hat{\mu})} \frac{\left(f_{\mu} \circ g_{\mu}^{-1}\right)\left(z \sqrt{h_{0}(\mu)}\right)}{(1-z)^{\left(\frac{\alpha}{\beta}\right)(\mu)}}=\left(a\left(2 h_{0} / b\right)^{\alpha / \beta}\right)(\hat{\mu}),
$$

and so the result follows. 
Lemma 3.12. Let $f$ be an analytic function on $[0,1), \nu_{1}, \nu_{2}, \ldots, \nu_{n} \in \mathbb{R}$ and $\ell \in \mathbb{N}$. Let us assume that $\mathscr{D}_{\boldsymbol{\nu}_{n-1}}[f]$ is quantifiable at 1 by $\xi$. If $\xi<3 / 2-\ell$, then

$$
N_{\ell}\left[\mathscr{D}_{\nu_{n}}[f]\right]=c_{n}\left(1-2 \ell-\nu_{n}\right) N_{\ell}\left[\mathscr{D}_{\boldsymbol{\nu}_{n-1}}[f]\right],
$$

where $c_{1}:=1$ and $c_{n}:=\prod_{i=1}^{n-1}\left(\nu_{n}-\nu_{i}\right)$ for $n \geqslant 2$.

Proof. Due to $f \in \mathscr{C}^{\omega}([0,1))$, from the definition of $\mathscr{B}$ it follows that $\mathscr{B}[f] \in \mathscr{C}^{\omega}([0,+\infty))$. By Lemma 3.9, the function $\left(\mathscr{B} \circ \mathscr{D}_{\boldsymbol{\nu}_{n-1}}\right)[f]$ is quantifiable at $+\infty$ by $2 \xi-2<1-2 \ell$. Thus, since $\mathscr{B} \circ \mathscr{D}_{\boldsymbol{\nu}_{n-1}}=\mathscr{L}_{\boldsymbol{\nu}_{n-1}} \circ \mathscr{B}$ by $(b)$ in Lemma 3.8 , by applying Lemma 2.15 we can assert that

$$
M_{\ell}\left[\left(\mathscr{L}_{\boldsymbol{\nu}_{n}} \circ \mathscr{B}\right)[f]\right]=c_{n}\left(1-2 \ell-\nu_{n}\right) M_{\ell}\left[\left(\mathscr{L}_{\boldsymbol{\nu}_{n-1}} \circ \mathscr{B}\right)[f]\right] .
$$

Now the result follows by using $(b)$ and $(d)$ in Lemma 3.8 .

The following is our main result in order to study the criticality of the outer boundary in case that its energy level is finite. As usual we point out that, in its statement, the assumptions requiring the existence of functions $\nu_{1}, \nu_{2}, \ldots, \nu_{n}$ for $n=0$ and that $N_{1} \equiv N_{2} \equiv \ldots \equiv N_{j-1} \equiv 0$ for $j=1$ are void.

Theorem B. Let $\left\{X_{\mu}\right\}_{\mu \in \Lambda}$ be a family of potential analytic systems verifying $(\mathbf{H})$ such that $h_{0}(\mu)<+\infty$ for all $\mu \in \Lambda$. Assume that there exist $n \geqslant 0$ continuous functions $\nu_{1}, \nu_{2}, \ldots, \nu_{n}$ in a neighbourhood of some fixed $\hat{\mu} \in \Lambda$ such that the family $\left\{\left(\mathscr{D}_{\boldsymbol{\nu}_{n}(\mu)} \circ \mathcal{P}\right)\left[z \sqrt{h_{0}(\mu)}\left(g_{\mu}^{-1}\right)^{\prime \prime}\left(z \sqrt{h_{0}(\mu)}\right)\right]\right\}_{\mu \in \Lambda}$ is continuously quantifiable in $\Lambda$ at $z=1$ by $\xi(\mu)$. For each $i \in \mathbb{N}$, let $N_{i}(\mu)$ be the $i$-th momentum of $\left(\mathscr{D}_{\boldsymbol{\nu}_{n}(\mu)} \circ \mathcal{P}\right)\left[z \sqrt{h_{0}(\mu)}\left(g_{\mu}^{-1}\right)^{\prime \prime}\left(z \sqrt{h_{0}(\mu)}\right)\right]$, whenever it is well defined. The following assertions hold:

(a) If $\xi(\hat{\mu})>\frac{1}{2}$, then $\operatorname{Crit}\left(\left(\Pi_{\hat{\mu}}, X_{\hat{\mu}}\right), X_{\mu}\right) \leqslant n$.

(b) If $\xi(\hat{\mu})<\frac{1}{2}$, let $m \in \mathbb{N}$ be such that $\xi(\hat{\mu})+m \in\left[\frac{1}{2}, \frac{3}{2}\right)$. Then $\operatorname{Crit}\left(\left(\Pi_{\hat{\mu}}, X_{\hat{\mu}}\right), X_{\mu}\right) \leqslant n$ in case that

(b1) either $N_{1} \equiv N_{2} \equiv \ldots \equiv N_{j-1} \equiv 0$ and $N_{j}(\hat{\mu}) \neq 0$ for some $j \in\{1,2, \ldots, m\}$,

(b2) or $N_{1} \equiv N_{2} \equiv \ldots \equiv N_{m} \equiv 0$ and $\xi(\hat{\mu})+m \notin\left\{\frac{1}{2}, 1\right\}$.

Finally, if the following conditions are verified, then $\left\{\left(\mathscr{D}_{\boldsymbol{\nu}_{n}(\mu)} \circ \mathcal{P}\right)\left[z \sqrt{h_{0}(\mu)}\left(g_{\mu}^{-1}\right)^{\prime \prime}\left(z \sqrt{h_{0}(\mu)}\right)\right]\right\}_{\mu \in \Lambda}$ is continuously quantifiable at $z=1$ by $\xi(\mu)=-\min \left\{\left(\frac{\alpha_{\ell}}{\beta_{\ell}}\right)(\mu),\left(\frac{\alpha_{r}}{\beta_{r}}\right)(\mu)\right\}-\frac{1}{2} \sum_{i=1}^{n} \nu_{i}(\mu)-\frac{n(n+1)}{2}+1$ :

(i) $\left\{h_{0}(\mu)-V_{\mu}\right\}_{\mu \in \Lambda}$ is continuously quantifiable at $x_{\ell}(\mu)$ by $\beta_{\ell}(\mu)$ and at $x_{r}(\mu)$ by $\beta_{r}(\mu)$ with limits $b_{\ell}(\mu)$ and $b_{r}(\mu)$, respectively,

(ii) setting $\mathscr{R}_{\mu}:=\frac{\left(V_{\mu}^{\prime}\right)^{2}-2 V_{\mu} V_{\mu}^{\prime \prime}}{\left(V_{\mu}^{\prime}\right)^{3}}$, the function

$$
x \longmapsto V_{\mu}^{\prime}(x)^{-\frac{n(n+1)}{2}} W\left[\left(\frac{V_{\mu}}{h_{0}(\mu)-V_{\mu}}\right)^{\frac{\nu_{1}(\mu)}{2}}, \ldots,\left(\frac{V_{\mu}}{h_{0}(\mu)-V_{\mu}}\right)^{\frac{\nu_{n}(\mu)}{2}},\left(h_{0}(\mu)-V_{\mu}\right) V_{\mu}^{\frac{1}{2}} \mathscr{R}_{\mu}\right](x)
$$

is continuously quantifiable at $x_{\ell}(\mu)$ by $\alpha_{\ell}(\mu)$ and at $x_{r}(\mu)$ by $\alpha_{r}(\mu)$ with limits $a_{\ell}(\mu)$ and $a_{r}(\mu)$, respectively,

(iii) and either $\frac{\alpha_{\ell}}{\beta_{\ell}}(\mu) \neq \frac{\alpha_{r}}{\beta_{r}}(\mu)$ or, otherwise, $\left(a_{r}\left(b_{r}\right)^{-\frac{\alpha_{r}}{\beta_{r}}}+(-1)^{\frac{n(n+1)}{2}} a_{\ell}\left(b_{\ell}\right)^{-\frac{\alpha_{\ell}}{\beta_{\ell}}}\right)(\mu) \neq 0$.

Proof. Set $f_{\mu}(z):=\mathcal{P}\left[z \sqrt{h_{0}(\mu)}\left(g_{\mu}^{-1}\right)^{\prime \prime}\left(z \sqrt{h_{0}(\mu)}\right)\right]$ for shortness. Then, the hypothesis $(\mathbf{H})$ and Lemma 3.3 guarantee that $\left\{f_{\mu}\right\}_{\mu \in \Lambda}$ is a continuous family of analytic functions on $[0,1)$. Furthermore, see (7), recall that $\sqrt{2} h^{2} T_{\mu}^{\prime}\left(h^{2}\right)=\mathscr{F}\left[f_{\mu}\right](h)$ for all $h \in\left(0, \sqrt{h_{0}(\mu)}\right)$. Hence the obvious rescaling yields to the identity

$$
\mathscr{F}\left[f_{\mu}\right](z)=\sqrt{2} h_{0}(\mu) z^{2} T_{\mu}^{\prime}\left(h_{0}(\mu) z^{2}\right), \text { for all } z \in(0,1) .
$$


So we must show that there exist $\varepsilon>0$ and a neighbourhood $U$ of $\hat{\mu}$ such that $\mathscr{F}\left[f_{\mu}\right](z)$ has at most $n$ zeros for $z \in(1-\varepsilon, 1)$, multiplicities taking into account, for all $\mu \in U$. Recall that, by $(b)$ in Lemma 3.8 , $\mathscr{B} \circ \mathscr{D}_{\boldsymbol{\nu}_{n}}=\mathscr{L}_{\boldsymbol{\nu}_{n}} \circ \mathscr{B}$. This will allow us to transfer the assumptions on the family $\left\{\mathscr{D}_{\boldsymbol{\nu}_{n}(\mu)}\left[f_{\mu}\right]\right\}_{\mu \in \Lambda}$, which is defined on $[0,1)$, to another family defined on $[0,+\infty)$ and then apply Proposition 2.16 as we did in the proof of Theorem A. With this aim in view we first note that

$$
\begin{aligned}
\left(\mathscr{L}_{\boldsymbol{\nu}_{n}(\mu)} \circ \mathscr{F} \circ \mathscr{B}\right)\left[f_{\mu}\right](x) & =\mathscr{L}_{\boldsymbol{\nu}_{n}(\mu)}\left[\sqrt{1+x^{2}}(\mathscr{B} \circ \mathscr{F})\left[f_{\mu}\right](x)\right] \\
& =\left(\mathscr{L}_{\boldsymbol{\nu}_{n}(\mu)} \circ \mathscr{B}\right)\left[\left(1-x^{2}\right)^{-\frac{1}{2}} \mathscr{F}\left[f_{\mu}\right](x)\right] \\
& =\left(\mathscr{B} \circ \mathscr{D}_{\boldsymbol{\nu}_{n}(\mu)}\right)\left[\left(1-x^{2}\right)^{-\frac{1}{2}} \mathscr{F}\left[f_{\mu}\right](x)\right],
\end{aligned}
$$

where we use $(c)$ in Lemma 3.8 in the first equality, the identity $\sqrt{1+x^{2}} \mathscr{B}[\phi](x)=\mathscr{B}\left[\left(1-x^{2}\right)^{-\frac{1}{2}} \phi(x)\right]$ with $\phi=\mathscr{F}\left[f_{\mu}\right]$ in the second one, and $(b)$ in Lemma 3.8 in the third one. Note that $\left\{\left(\mathscr{L}_{\boldsymbol{\nu}_{n}(\mu)} \circ \mathscr{F}_{\circ} \circ \mathscr{B}\right)\left[f_{\mu}\right]\right\}_{\mu \in \Lambda}$ is a continuous family of analytic functions on $[0,+\infty)$.

We claim that if $\left\{\left(\mathscr{L}_{\boldsymbol{\nu}_{n}(\mu)} \circ \mathscr{F} \circ \mathscr{B}\right)\left[f_{\mu}\right]\right\}_{\mu \in \Lambda}$ is continuously quantifiable at $+\infty$ in $\hat{\mu}$, then the criticality of $X_{\mu}$ at the outer boundary of the period annulus is at most $n$. Indeed, to show this suppose that the quantifier is $\eta(\mu)$. Then, on account of the previous equality and Lemma $3.9,\left\{\mathscr{D}_{\boldsymbol{\nu}_{n}(\mu)}\left[\left(1-x^{2}\right)^{-\frac{1}{2}} \mathscr{F}\left[f_{\mu}\right](x)\right]\right\}_{\mu \in \Lambda}$ is continuously quantifiable at $z=1$ in $\hat{\mu}$ by $\frac{1}{2} \eta(\mu)+1$, i.e.

$$
\lim _{(x, \mu) \rightarrow(1, \hat{\mu})}(1-x)^{\frac{1}{2} \eta(\mu)+1} \mathscr{D}_{\boldsymbol{\nu}_{n}(\mu)}\left[\left(1-x^{2}\right)^{-\frac{1}{2}} \mathscr{F}\left[f_{\mu}\right](x)\right] \neq 0 .
$$

Thus, according to the definition of $\mathscr{D}_{\boldsymbol{\nu}_{n}}$ in $(9)$,

$$
\lim _{(x, \mu) \rightarrow(1, \hat{\mu})}(1-x)^{\frac{\eta(\mu)}{2}+1}\left(x\left(1-x^{2}\right)\right)^{\frac{n(n+1)}{2}} \frac{W\left[\psi_{\nu_{1}(\mu)}(x), \ldots, \psi_{\nu_{n}(\mu)}(x),\left(1-x^{2}\right)^{-\frac{1}{2}} \mathscr{F}\left[f_{\mu}\right](x)\right]}{\prod_{i=1}^{n} \psi_{\nu_{i}(\mu)}(x)} \neq 0,
$$

which, due to $\psi_{\nu}(x)=\frac{1}{1-x^{2}}\left(\frac{x}{\sqrt{1-x^{2}}}\right)^{\nu}$, easily implies that

$$
\lim _{(x, \mu) \rightarrow(1, \hat{\mu})}(1-x)^{\kappa(\mu)} W\left[\psi_{\nu_{1}(\mu)}(x), \ldots, \psi_{\nu_{n}(\mu)}(x),\left(1-x^{2}\right)^{-\frac{1}{2}} \mathscr{F}\left[f_{\mu}\right](x)\right] \neq 0,
$$

where $\kappa(\mu):=\frac{1}{2}\left(\eta(\mu)+(n+1)(n+2)+\sum_{i=1}^{n} \nu_{i}(\mu)\right)$. Now the claim follows by applying Lemma 3.10 and taking (12) into account.

We are now in position to prove $(a)$ and $(b)$ in the first part of the statement. To this end recall that, by assumption, the family $\left\{\mathscr{D}_{\boldsymbol{\nu}_{n}(\mu)}\left[f_{\mu}\right]\right\}_{\mu \in \Lambda}$ is continuously quantifiable in $\Lambda$ at $z=1$ by $\xi(\mu)$. On the other hand, by $(b)$ in Lemma 3.8, $\left(\mathscr{B} \circ \mathscr{D}_{\boldsymbol{\nu}_{n}(\mu)}\right)\left[f_{\mu}\right]=\left(\mathscr{L}_{\boldsymbol{\nu}_{n}(\mu)} \circ \mathscr{B}\right)\left[f_{\mu}\right]$. Hence by applying Lemma 3.9 we can assert that the family $\left\{\left(\mathscr{L}_{\boldsymbol{\nu}_{n}(\mu)} \circ \mathscr{B}\right)\left[f_{\mu}\right]\right\}_{\mu \in \Lambda}$ is continuously quantifiable in $\Lambda$ at $+\infty$ by $2 \xi(\mu)-2$. (This is precisely the family defined on $[0,+\infty)$ that in the beginning of the proof we refer to.)

- If $\xi(\hat{\mu})>\frac{1}{2}$, then $2 \xi(\hat{\mu})-2>-1$ and so Proposition 2.16 applied to $\left\{\mathscr{B}\left[f_{\mu}\right]\right\}_{\mu \in \Lambda}$ guarantees that $\left\{\left(\mathscr{L}_{\boldsymbol{\nu}_{n}(\mu)} \circ \mathscr{F} \circ \mathscr{B}\right)\left[f_{\mu}\right]\right\}_{\mu \in \Lambda}$ is continuously quantifiable in a neighbourhood of $\hat{\mu}$ at $+\infty$ by $2 \xi(\mu)-2$. This, thanks to the previous claim, proves that $\operatorname{Crit}\left(\left(\Pi_{\hat{\mu}}, X_{\hat{\mu}}\right), X_{\mu}\right) \leqslant n$ and hence $(a)$ follows.

- To show $(b)$ we use that, by $(b)$ and $(d)$ in Lemma 3.8,

$$
N_{\ell}\left[\mathscr{D}_{\boldsymbol{\nu}_{n}(\mu)}\left[f_{\mu}\right]\right]=M_{\ell}\left[\left(\mathscr{B} \circ \mathscr{D}_{\boldsymbol{\nu}_{n}(\mu)}\right)\left[f_{\mu}\right]\right]=M_{\ell}\left[\left(\mathscr{L}_{\boldsymbol{\nu}_{n}(\mu)} \circ \mathscr{B}\right)\left[f_{\mu}\right]\right]
$$

Then the result follows straightforward by applying Proposition 2.16 and taking the previous claim into account again.

Let us turn next to the proof of the second part of the result. Denote $\Delta(\mu):=\sum_{i=1}^{n}\left(\nu_{i}(\mu)-i\right)$ for the sake shortness. For the same reason, from now on we omit the dependence on $\mu$ when it is not essential. That being said note that, due to $V_{\mu}\left(g_{\mu}^{-1}(z)\right)=z^{2}$,

$$
\psi_{\nu_{i}}(z)=\left(\frac{h_{0}}{h_{0}-V_{\mu}}\left(\frac{V_{\mu}}{h_{0}-V_{\mu}}\right)^{\frac{\nu_{i}}{2}}\right)\left(g_{\mu}^{-1}\left(z \sqrt{h_{0}}\right)\right) \text { for all } z \in(0,1) .
$$


In addition, since $\left(g_{\mu}^{-1}(z)\right)^{\prime \prime}=2 \mathscr{R}_{\mu}\left(g_{\mu}^{-1}(z)\right)$, we have that $f_{\mu}$ is the even part of $2\left(g_{\mu} \mathscr{R}_{\mu}\right)\left(g_{\mu}^{-1}\left(z \sqrt{h_{0}}\right)\right)$. Consequently, taking Lemma 2.11 also into account, some computations show that

$$
\mathscr{D}_{\boldsymbol{\nu}_{n}(\mu)}\left[z \sqrt{h_{0}}\left(g_{\mu}^{-1}\right)^{\prime \prime}\left(z \sqrt{h_{0}}\right)\right]=2^{1+\frac{n(n+1)}{2}} h_{0}^{-\frac{n(n+1)}{2}} S_{\mu}\left(g_{\mu}^{-1}\left(z \sqrt{h_{0}}\right)\right) \text {, for all } z \in(0,1),
$$

where

$$
S_{\mu}(x):=\frac{W\left[\left(\frac{V_{\mu}}{h_{0}-V_{\mu}}\right)^{\frac{\nu_{1}}{2}}, \ldots,\left(\frac{V_{\mu}}{h_{0}-V_{\mu}}\right)^{\frac{\nu_{n}}{2}},\left(h_{0}-V_{\mu}\right) V_{\mu}^{\frac{1}{2}} \mathscr{R}_{\mu}\right](x)}{\left(h_{0}-V_{\mu}(x)\right)^{-\frac{\Delta}{2}-\frac{3 n(n+1)}{4}+1} V_{\mu}(x)^{-\frac{n(n+1)}{4}+\frac{\Delta}{2}}\left(V_{\mu}^{\prime}(x)\right)^{\frac{n(n+1)}{2}}} .
$$

Similarly, due to $g(z)=-\sqrt{V(z)}$ for $z<0$, we have that

$$
\mathscr{D}_{\boldsymbol{\nu}_{n}(\mu)}\left[-z \sqrt{h_{0}}\left(g_{\mu}^{-1}\right)^{\prime \prime}\left(-z \sqrt{h_{0}}\right)\right]=-(-2)^{1+\frac{n(n+1)}{2}} h_{0}^{-\frac{n(n+1)}{2}} S_{\mu}\left(g_{\mu}^{-1}\left(-z \sqrt{h_{0}}\right)\right) \text {, for all } z \in(0,1) .
$$

On account of the assumptions in $(i)$ and $(i i)$, we can assert that $\left\{S_{\mu}\right\}_{\mu \in \Lambda}$ is continuously quantifiable at $x_{\ell}$ by $\gamma_{\ell}:=\alpha_{\ell}+\beta_{\ell}\left(\frac{\Delta}{2}+\frac{3 n(n+1)}{4}-1\right)$ and at $x_{r}$ by $\gamma_{r}:=\alpha_{r}+\beta_{r}\left(\frac{\Delta}{2}+\frac{3 n(n+1)}{4}-1\right)$, with limits $c_{\ell}:=$ $a_{\ell} b_{\ell} \frac{\Delta}{2}+\frac{3 n(n+1)}{4}-1 h_{0} \frac{n(n+1)}{4}-\frac{\Delta}{2}$ and $c_{r}:=a_{r} b_{r}^{\frac{\Delta}{2}+\frac{3 n(n+1)}{4}-1} h_{0} \frac{n(n+1)}{4}-\frac{\Delta}{2}$, respectively. Then, by Lemma 3.11, some computations show that $\left\{\left(S_{\mu} \circ g_{\mu}^{-1}\right)\left(z \sqrt{h_{0}}\right)\right\}_{\mu \in \Lambda}$ is continuously quantifiable at $z=-1$ by $-\frac{\gamma_{\ell}}{\beta_{\ell}}$ and at $z=1$ by $-\frac{\gamma_{r}}{\beta_{r}}$, with limits $c_{\ell}\left(\frac{2 h_{0}}{b_{\ell}}\right)^{\frac{\gamma_{\ell}}{\beta_{\ell}}}$ and $c_{r}\left(\frac{2 h_{0}}{b_{r}}\right)^{\frac{\gamma_{r}}{\beta_{r}}}$, respectively. Accordingly, by the assumption in $($ iii $)$, we have that $\left\{\left(\mathscr{D}_{\boldsymbol{\nu}_{n}(\mu)} \circ \mathcal{P}\right)\left[z \sqrt{h_{0}}\left(g_{\mu}^{-1}\right)^{\prime \prime}\left(z \sqrt{h_{0}}\right)\right]\right\}_{\mu \in \Lambda}$ is continuously quantifiable at $z=1$ by $\xi=\max \left\{-\frac{\alpha_{\ell}}{\beta_{\ell}},-\frac{\alpha_{r}}{\beta_{r}}\right\}-\frac{\Delta}{2}-\frac{3 n(n+1)}{4}+1$. This shows the second assertion and completes the proof.

\section{Application}

In this section we resume the study that we began in [13] for the family of potential differential systems $\left\{X_{\mu}\right\}_{\mu \in \Lambda}$, where

$$
X_{\mu}=-y \partial_{x}+\left((x+1)^{p}-(x+1)^{q}\right) \partial_{y} \text { with } \mu=(q, p)
$$

and $\Lambda=\left\{(q, p) \in \mathbb{R}^{2}: p>q\right\}$. Following the previous notation, we define

$$
V_{\mu}(x)=\int_{1}^{x+1}\left(u^{p}-u^{q}\right) d u
$$

We will prove Theorem $\mathrm{C}$ to illustrate the application of the theoretical results we have obtained so far. Before we need to show several lemmas. The first one will in particular ensure the uniqueness of $p_{0}$ as introduced in the statement of Theorem C.

Lemma 4.1. (a) The function $f(p)=(2+2 p)^{\frac{2+2 p}{1+2 p}}-2(1+2 p)$ has a unique zero on $\left(-\frac{1}{2},+\infty\right)$.

(b) If $p \in\left(-\frac{1}{2},+\infty\right)$, then $(2+2 p)^{\frac{2+2 p}{1+2 p}}+4 p(1+2 p) \neq 0$.

Proof. In order to prove $(a)$ we claim that $g(x):=x^{\frac{x}{x-1}}-2 x+2$ is monotonous decreasing on $(1,+\infty)$. Note that $g(x)=f(x / 2-1)$ and, consequently, $(a)$ will follow once we prove the claim because one can easily verify that $\lim _{x \rightarrow 1} g(x)=e$ and $\lim _{x \rightarrow+\infty} g(x)=-\infty$. To show the claim we first note that

$$
g^{\prime}(x)=-2+\frac{x^{\frac{x}{x-1}}(x-1-\log (x))}{(x-1)^{2}} \text { and } g^{\prime \prime}(x)=\frac{x^{\frac{x}{x-1}}\left(x \log (x)^{2}-(x-1)^{2}\right)}{(x-1)^{4}}
$$

Since $\lim _{x \rightarrow 1} g^{\prime}(x)<0$, it suffices to show that $g^{\prime \prime}(x)<0$ for all $x \in(1,+\infty)$, which is equivalent to $\kappa(x):=x \log (x)^{2}-(x-1)^{2}<0$. However this is clear because one can verify that $\kappa(1)=\kappa^{\prime}(1)=\kappa^{\prime \prime}(1)=0$ and $\kappa^{\prime \prime \prime}(x)=-\frac{2 \log (x)}{x^{2}}<0$ for all $x>1$. This shows the validity of $(a)$. The proof of $(b)$ follows similarly and we omit it here for the sake of brevity. 
Definition 4.2. The function defined by

$$
{ }_{2} F_{1}(a, b, c ; z)=\sum_{n=0}^{\infty} \frac{(a)_{n}(b)_{n}}{(c)_{n}} \frac{z^{n}}{n !},
$$

where $(k)_{n}:=k(k+1) \ldots(k+n-1)$ for $n \geqslant 1$ and $(k)_{0}:=1$, is the Gaussian Hypergeometric function.

Lemma 4.3. If $a, b \in \mathbb{C}$, then $\frac{d}{d z} 2 F_{1}(a, b, b+1 ; z)=\frac{b}{z}\left((1-z)^{-a}-{ }_{2} F_{1}(a, b, b+1 ; z)\right)$.

Proof. This is straightforward by using the formulae in [1]. Indeed, it shows that

$$
\frac{d}{d z} z_{2}^{b} F_{1}(a, b, b+1 ; z)=b z^{b-1}{ }_{2} F_{1}(a, b, b ; z)=b z^{b-1}(1-z)^{-a},
$$

where the first equality is a particular case of 15.2.4 and the second one follows by applying 15.1.8. Then an easy manipulation yields to the desired equality after deriving the product on the left.

For the parameter values under consideration in Theorem C, it is easy to show that the projection of the period annulus on the $x$-axis is $\mathcal{I}_{\mu}=(-1, \rho(\mu))$, with

$$
\rho(\mu):=\left(\frac{p+1}{q+1}\right)^{\frac{1}{p-q}}-1,
$$

and that the energy level at the outer boundary is $h_{0}(\mu):=\frac{p-q}{(p+1)(q+1)}$. Then, with notation introduced in Section 3.2 , we have the following result:

Lemma 4.4. Let $\left\{X_{\mu}\right\}_{\mu \in \Lambda}$ be the family of potential vector fields in (1) and let $f_{\mu}$ be the even part of $z \longmapsto z \sqrt{h_{0}(\mu)}\left(g_{\mu}^{-1}\right)^{\prime \prime}\left(z \sqrt{h_{0}(\mu)}\right)$, where $g_{\mu}(x):=\operatorname{sgn}(x) \sqrt{V_{\mu}(x)}$ for $x \in \mathcal{I}_{\mu}$. Then the following hold:

(a) If $\mu=\left(-\frac{1}{2}, p\right)$ with $p \in\left(-\frac{1}{2},+\infty\right) \backslash\{0\}$, then $N_{1}\left[f_{\mu}\right] \neq 0$.

(b) If $\mu=(0, p)$ with $p \in\left(\frac{1}{2},+\infty\right) \backslash\{1\}$ and $\nu_{1}(\mu)=0$, then $N_{1}\left[\mathscr{D}_{\nu_{1}(\mu)}\left[f_{\mu}\right]\right] \neq 0$.

Proof. For the sake of shortness we shall omit the nonessential dependence on $\mu$. That being said, the change of variable $x=g^{-1}\left(z \sqrt{h_{0}}\right)$ gives formally

$$
N_{1}[f]=\int_{-1}^{1} \frac{z \sqrt{h_{0}}\left(g^{-1}\right)^{\prime \prime}\left(z \sqrt{h_{0}}\right)}{\sqrt{1-z^{2}}} d z=\int_{-1}^{\rho} \frac{\left(V^{\prime 2}-2 V V^{\prime \prime}\right)(x)}{V^{\prime}(x)^{2} \sqrt{h_{0}-V(x)}} d x .
$$

It is proved in [13, Lemma A.4] that the second integral is convergent for $p>q$ and $q \in\left(-1,-\frac{1}{3}\right)$. That result provides moreover its precise value in terms of the Gamma function. On account of this we can assert that, for those parameters,

$$
N_{1}[f]=-\sqrt{\pi} \frac{(p+1)^{\frac{1}{2}}(p+2 q+1)}{(p-q)(q+1)(\rho(\mu)+1)^{\frac{3 p+1}{2}}} \frac{\Gamma\left(-\frac{3 q+1}{2(p-q)}\right)}{\Gamma\left(\frac{p-4 q-1}{2(p-q)}\right)} .
$$

Therefore, for $\mu=\left(-\frac{1}{2}, p\right)$ with $p>-\frac{1}{2}, N_{1}[f]=0$ if and only if $p=0$. This proves $(a)$.

In order to show $(b)$ let us take $\mu=(0, p)$ with $p>\frac{1}{2}$. We shall take advantage of the second part of Theorem B with $n=0$ to prove that then $f$ is quantifiable at $z=1$ by $\xi<\frac{1}{2}$. Indeed, if $\mu=(0, p)$ then $h_{0}-V(x)=x+1-\frac{(x+1)^{p+1}}{p+1}$ is quantifiable at $x_{\ell}=-1$ by $\beta_{\ell}=-1$ and at $x_{r}=(p+1)^{\frac{1}{p}}-1$ by $\beta_{r}=-1$. On the other hand, some computations show that $\left(h_{0}-V(x)\right) V(x)^{\frac{1}{2}} \mathscr{R}(x)$ is quantifiable at $x_{\ell}=-1$ by $\alpha_{\ell}=-1$ if $p>1$ and by $\alpha_{\ell}=-p$ if $p<1$, in both cases with limit $a_{\ell}=\sqrt{h_{0}}$. Similarly, it is quantifiable at $x_{r}=(p+1)^{\frac{1}{p}}-1$ by $\alpha_{r}=-1$ with limit $a_{r}=\sqrt{h_{0}}\left(1-2(1+p)^{-\frac{1}{p}}\right)$. According to this and taking $p>\frac{1}{2}$ into account, by applying the second part of Theorem B with $n=0$, it turns out that $f$ is quantifiable at $z=1$ by 
$\xi<\frac{1}{2}$. Then, by applying Lemma 3.12 with $\ell=n=1$ and $\nu_{1}=0, N_{1}\left[\mathscr{D}_{\nu_{1}}[f]\right]=-\left(1+\nu_{1}\right) N_{1}[f]=-N_{1}[f]$. Consequently, from (13),

$$
N_{1}\left[\mathscr{D}_{\nu_{1}}[f]\right]=-N_{1}[f]=-\int_{-1}^{\rho} \frac{\left(V^{\prime 2}-2 V V^{\prime \prime}\right)(x)}{V^{\prime}(x)^{2} \sqrt{h_{0}-V(x)}} d x .
$$

Since $V(x)=\frac{(x+1)^{p+1}-1}{p+1}-x$ for $\mu=(0, p)$ with $p \neq-1$, some long but easy computations by applying Lemma 4.3 show that

$$
G(x):=2 \frac{{ }_{2} F_{1}\left(\frac{1}{2},-\frac{1}{2 p}, 1-\frac{1}{2 p}, \frac{(x+1)^{p}}{p+1}\right)-\frac{x}{(x+1)^{p}-1} \sqrt{1-\frac{(x+1)^{p}}{1+p}}}{\sqrt{x+1}}
$$

is a primitive of $\frac{V^{\prime 2}-2 V V^{\prime}}{V^{\prime 2} \sqrt{h_{0}-V}}$. By $[1,15.1 .20]$, and using $p>\frac{1}{2}$, we get that $\lim _{x \rightarrow \rho} G(x)=\frac{2 \sqrt{\pi} \Gamma\left(1-\frac{1}{2 p}\right)}{(p+1)^{\frac{1}{2 p}} \Gamma\left(\frac{p-1}{2 p}\right)}$. On the other hand, since by definition

$$
{ }_{2} F_{1}\left(\frac{1}{2},-\frac{1}{2 p}, 1-\frac{1}{2 p}, \frac{(x+1)^{p}}{p+1}\right)=1-\frac{(x+1)^{p}}{2(2 p-1)(p+1)}+\mathrm{o}\left((x+1)^{p}\right),
$$

we can assert, taking $p>\frac{1}{2}$ into account once again, that $\lim _{x \rightarrow-1} G(x)=0$. Consequently,

$$
N_{1}\left[\mathscr{D}_{\nu_{1}}[f]\right]=-N_{1}[f]=\lim _{x \rightarrow-1} G(x)-\lim _{x \rightarrow \rho} G(x)=\frac{-2 \sqrt{\pi} \Gamma\left(1-\frac{1}{2 p}\right)}{(p+1)^{\frac{1}{2 p}} \Gamma\left(\frac{p-1}{2 p}\right)},
$$

which shows that if $p \in\left(\frac{1}{2},+\infty\right) \backslash\{1\}$ and $\nu_{1}=0$, then $N_{1}\left[\mathscr{D}_{\nu_{1}}[f]\right] \neq 0$, as desired.

Proof of Theorem C. Note first that if $p$ and $q$ are both different from -1 , then

$$
V_{\mu}(x)=\frac{(x+1)^{p+1}}{p+1}-\frac{(x+1)^{q+1}}{q+1}+h_{0}(\mu),
$$

where $h_{0}(\mu):=\frac{p-q}{(p+1)(q+1)}$ is the energy level at the outer boundary of $\mathscr{P}_{\mu}$. Moreover, the projection of $\mathscr{P}_{\mu}$ on the $x$-axis is $\mathcal{I}_{\mu}=\left(x_{\ell}(\mu), x_{r}(\mu)\right)$, with

$$
x_{\ell}(\mu)=-1 \text { and } x_{r}(\mu)=\rho(\mu):=\left(\frac{p+1}{q+1}\right)^{\frac{1}{p-q}}-1 .
$$

Following the notation in Theorem B, from (14) and on account $p>q$, we have that $\left\{h_{0}(\mu)-V_{\mu}(x)\right\}_{\mu \in \Lambda}$ is continuously quantifiable for any $\hat{\mu}=(\hat{q}, \hat{p}) \in \Lambda$ at $x=x_{\ell}$ by $\beta_{\ell}(\mu)$ with limit $b_{\ell}$ and at $x=x_{r}$ by $\beta_{r}(\mu)$ with limit $b_{r}$, where

$$
\beta_{\ell}(\mu)=-(q+1), \beta_{r}(\mu)=-1, b_{\ell}=\frac{1}{\hat{q}+1} \text { and } b_{r}=V_{\hat{\mu}}^{\prime}\left(x_{r}\right)
$$

We will prove $(a)$ by applying Theorem B with $n=0$. So let us consider $\hat{\mu}=(\hat{q}, \hat{p})$ with $\hat{q}=-\frac{1}{2}$ and $\hat{p} \in\left(-\frac{1}{2},+\infty\right) \backslash\left\{0, p_{0}\right\}$, where $p_{0}$ is the unique root of $f(p)=0$ on $\left(-\frac{1}{2},+\infty\right)$. We begin by studying the quantifiers of the family $\left\{\left(h_{0}-V_{\mu}\right) V_{\mu}^{\frac{1}{2}} \mathscr{R}_{\mu}\right\}_{\mu \in \Lambda}$, where recall that $\mathscr{R}_{\mu}:=\frac{\left(V_{\mu}^{\prime}\right)^{2}-2 V_{\mu} V_{\mu}^{\prime \prime}}{\left(V_{\mu}^{\prime}\right)^{3}}$. An easy computation from (14) shows that this family is continuously quantifiable in $\hat{\mu}$ at $x=x_{\ell}$ by $\alpha_{\ell}(\mu)=q$ with limit $a_{\ell}=2\left(2-\frac{1}{\hat{p}+1}\right)^{\frac{3}{2}}$. Note on the other hand that $V_{\mu}$ is analytic at $x=x_{r}$ with $V_{\hat{\mu}}^{\prime}\left(x_{r}\right) \neq 0$. Consequently the family is continuously quantifiable in $\hat{\mu}$ at $x=x_{r}$ by $\alpha_{r}(\mu)=-1$ with limit

$$
a_{r}=\sqrt{h_{0}(\hat{\mu})} \frac{V_{\hat{\mu}}^{\prime}\left(x_{r}\right)^{2}-2 h_{0}(\hat{\mu}) V_{\hat{\mu}}^{\prime \prime}\left(x_{r}\right)}{V_{\hat{\mu}}^{\prime}\left(x_{r}\right)^{2}}
$$


provided that $V_{\hat{\mu}}^{\prime}\left(x_{r}\right)^{2}-2 h_{0}(\hat{\mu}) V_{\hat{\mu}}^{\prime \prime}\left(x_{r}\right) \neq 0$. One can check that this is equivalent to require that $f(\hat{p}) \neq 0$, which is indeed satisfied because $\hat{p} \neq p_{0}$ by assumption, cf. (a) in Lemma 4.1. Accordingly, taking (15) also into account, we have that $\left(\frac{\alpha_{\ell}}{\beta_{\ell}}\right)(\hat{\mu})=\left(\frac{\alpha_{r}}{\beta_{r}}\right)(\hat{\mu})=1$ and

$$
\left(a_{r}\left(b_{r}\right)^{-\frac{\alpha_{r}}{\beta_{r}}}+a_{\ell}\left(b_{\ell}\right)^{-\frac{\alpha_{\ell}}{\beta_{\ell}}}\right)(\hat{\mu})=\frac{(2+2 \hat{p})^{\frac{2+2 p}{1+2 \hat{p}}}+4 \hat{p}(1+2 \hat{p})}{2(1+\hat{p})^{\frac{3}{2}} \sqrt{1+2 \hat{p}}} \neq 0 .
$$

(The fact that this expression is different from zero follows by (b) in Lemma 4.1.) Accordingly, by the second part of Theorem B, the family $\left\{\mathcal{P}\left[z \sqrt{h_{0}(\mu)}\left(g_{\mu}^{-1}\right)^{\prime \prime}\left(z \sqrt{h_{0}(\mu)}\right)\right]\right\}_{\mu \in \Lambda}$ is continuously quantifiable in $\hat{\mu}$ at $z=1$ by $\xi(\mu)=\max \left\{\frac{q}{q+1},-1\right\}+1$. We apply next the first part of Theorem B. To this end note that $\xi(\hat{\mu})=0$ and that, by $(a)$ in Lemma 4.4 , the first momentum of the even part of $z \longmapsto z \sqrt{h_{0}(\hat{\mu})}\left(g_{\hat{\mu}}^{-1}\right)^{\prime}\left(z \sqrt{h_{0}(\hat{\mu})}\right)$ does not vanish. Then, the application of $(b 1)$ in Theorem B with $n=0$ and $j=1$ shows that $\operatorname{Crit}\left(\left(\Pi_{\hat{\mu}}, X_{\hat{\mu}}\right), X_{\mu}\right)=0$. This proves the validity of $(a)$.

Finally let us turn to the proof of $(b)$. So consider now $\hat{\mu}=(\hat{q}, \hat{p})$ with $\hat{q}=0$ and $\hat{p} \in(0,+\infty) \backslash\left\{\frac{1}{2}, 1\right\}$. We note that, by $\left[13\right.$, Theorem E], $\operatorname{Crit}\left(\left(\Pi_{\hat{\mu}}, X_{\hat{\mu}}\right), X_{\mu}\right) \geqslant 1$, and so the result will follow by applying Theorem B with $n=1$. To this end we need to study the function

$$
\Psi_{\mu}(x):=\frac{1}{V_{\mu}^{\prime}(x)} W\left[\left(\frac{V_{\mu}}{h_{0}(\mu)-V_{\mu}}\right)^{\frac{\nu_{1}(\mu)}{2}},\left(h_{0}(\mu)-V_{\mu}\right) V_{\mu}^{\frac{1}{2}} \mathscr{R}_{\mu}\right](x),
$$

where $\nu_{1}$ is a continuous function to be determined. Some tedious calculations show that

$$
\Psi_{\mu}=\frac{\psi_{\mu}}{2 V_{\mu}^{\frac{1}{2}} V_{\mu}^{\prime 5}}\left(\frac{V_{\mu}}{h_{0}(\mu)-V_{\mu}}\right)^{\frac{\nu_{1}(\mu)}{2}},
$$

where, omitting the dependence on $\mu$ for shortness,

$$
\psi:=-\left(V^{\prime 2}-2 V V^{\prime \prime}\right)\left(V^{\prime 2}\left(h_{0}\left(\nu_{1}-1\right)+3 V\right)+6\left(h_{0}-V\right) V V^{\prime \prime}\right)+4 V^{2}\left(V-h_{0}\right) V^{\prime} V^{\prime \prime \prime} .
$$

By means of an algebraic manipulator we can assert that $\psi_{\mu}(x)$ is the sum of 15 monomials of the form $c(\mu)(x+1)^{n_{1} p+n_{2} q+n_{3}}$ with $n_{i} \in \mathbb{Z}$, for $i=1,2,3$, and $c$ a well defined rational function at $\mu=\hat{\mu}$. Moreover the monomial with the smallest exponent for $\mu \approx \hat{\mu}$ is $(x+1)^{3 q-1}$. Consequently,

$$
\psi_{\mu}(x)=(x+1)^{3 q-1}\left(\frac{2 q(p-q)^{2}\left(2 q-(q+1) \nu_{1}\right)}{(p+1)^{2}(q+1)^{2}}+r_{\mu}(x)\right),
$$

with $\lim _{x \rightarrow-1} r_{\mu}(x)=0$ uniformly for $\mu \approx \hat{\mu}$. This leads us to the choice $\nu_{1}(\mu)=\frac{2 q}{q+1}$, otherwise $\left\{\Psi_{\mu}\right\}_{\mu \in \Lambda}$ would not be continuously quantifiable in $\hat{\mu}$ at $x=x_{\ell}$. From now on we set $\nu_{1}(\mu):=\frac{2 q}{q+1}$. Accordingly, the monomial $(x+1)^{3 q-1}$ "disappears" and we get that

$$
\psi_{\mu}(x)=\frac{2(p-q)(q-1)}{(p+1)(q+1)^{3}}(x+1)^{4 q}+\frac{4(p-2 q)(p-q)^{3}(1+p-q)}{(p+1)^{3}(q+1)^{3}}(x+1)^{p+2 q-1}+\hat{r}_{\mu}(x)
$$

with both, $(x+1)^{-4 q} \hat{r}_{\mu}(x)$ and $(x+1)^{1-p-2 q} \hat{r}_{\mu}(x)$ tending to zero as $x \longrightarrow-1$ uniformly for $\mu \approx \hat{\mu}$. We now consider two cases, $\hat{p} \in(0,1)$ and $\hat{p}>1$.

- If $\hat{p} \in(0,1)$, then the monomial with smallest exponent in $\psi_{\mu}$ for $\mu \approx \hat{\mu}$ is $(x+1)^{p+2 q-1}$. Taking this into account, some computations show that the family $\left\{\Psi_{\mu}\right\}_{\mu \in \Lambda}$ is continuously quantifiable in $\hat{\mu}$ at $x=x_{\ell}$ by $\alpha_{\ell}(\mu)=1-p+4 q$.

- If $\hat{p}>1$, then $(x+1)^{4 q}$ is the monomial with smallest exponent in $\psi_{\mu}$ for $\mu \approx \hat{\mu}$ and, similarly as before, $\left\{\Psi_{\mu}\right\}_{\mu \in \Lambda}$ is continuously quantifiable in $\hat{\mu}$ at $x=x_{\ell}$ by $\alpha_{\ell}(\mu)=2 q$ with limit $a_{\ell}=\sqrt{\frac{\hat{p}}{\hat{p}+1}}$. 
On the other hand, taking advantage of the analyticity of $V_{\mu}$ at $x=x_{r}$, one can easily check that $\left\{\Psi_{\mu}\right\}_{\mu \in \Lambda}$ is continuously quantifiable in $\hat{\mu}$ at $x=x_{r}$ by $\alpha_{r}(\mu)=-\frac{q}{q+1}$ with limit $a_{r}=\hat{p}^{-\frac{1}{2}}(\hat{p}+1)^{-\frac{\hat{p}+2}{2 \hat{p}}}\left(2-(\hat{p}+1)^{\frac{1}{\hat{p}}}\right)$.

We are now in position to conclude the proof. Let us consider the case $\hat{p} \in(0,1)$ first. Then, on account of (15) and the values of $\alpha_{\ell}$ and $\alpha_{r}$ obtained above, by applying the second part of Theorem B with $n=1$ we get $\xi(\hat{\mu})=-\min \{\hat{p}-1,0\}=1-\hat{p}$. Consequently if $\hat{p} \in\left(0, \frac{1}{2}\right)$, then $\xi(\hat{\mu})>\frac{1}{2}$ and by $(a)$ in Theorem $\mathrm{B}$ we can assert that $\operatorname{Crit}\left(\left(\Pi_{\hat{\mu}}, X_{\hat{\mu}}\right), X_{\mu}\right) \leqslant 1$, as desired. If $\hat{p} \in\left(\frac{1}{2}, 1\right)$, then by ${ }^{(b)}$ in Lemma 4.4 we have $N_{1}(\hat{\mu}) \neq 0$ and hence, by $(b 1)$ in Theorem B, we get $\operatorname{Crit}\left(\left(\Pi_{\hat{\mu}}, X_{\hat{\mu}}\right), X_{\mu}\right) \leqslant 1$, as well. Let us consider finally the case $\hat{p}>1$. Then, from (15) and the values of $\alpha_{\ell}$ and $\alpha_{r}$ obtained above, $\left(\frac{\alpha_{\ell}}{\beta_{\ell}}\right)(\hat{\mu})=\left(\frac{\alpha_{r}}{\beta_{r}}\right)(\hat{\mu})=0$ and

$$
\left(a_{r}\left(b_{r}\right)^{-\frac{\alpha_{r}}{\beta_{r}}}-a_{\ell}\left(b_{\ell}\right)^{-\frac{\alpha_{\ell}}{\beta_{\ell}}}\right)(\hat{\mu})=\frac{(\hat{p}+1)^{-\frac{2+\hat{p}}{2 \hat{p}}}\left(2-(\hat{p}+1)^{\frac{\hat{p}+1}{\hat{p}}}\right)}{\sqrt{\hat{p}}} \neq 0
$$

for all $\hat{p}>1$. Thus, by the second part of Theorem B, $\xi(\hat{\mu})=0$. Since $N_{1}(\hat{\mu}) \neq 0$ due to $(b)$ in Lemma 4.4, by the first part of Theorem B we get $\operatorname{Crit}\left(\left(\Pi_{\hat{\mu}}, X_{\hat{\mu}}\right), X_{\mu}\right) \leqslant 1$ also in this case. This proves the result.

\section{References}

[1] M. Abramowitz, I. Stegun, Handbook of mathematical functions with formulas, graphs, and mathematical tables, 1992.

[2] T. Blows, N. Lloyd, The number of limit cycles of certain polynomial differential equations, Proc. Roy. Soc. Edinburgh Sect. A. 98 (1984) 215-239.

[3] C. Chicone, The monotonicity of the period function for planar hamiltonian vector fields, J. Differential Equations 69 (1987) 310-321.

[4] C. Chicone, Geometric methods of two-point nonlinear boundary value problem, J. Differential Equations 72 (1988) 360-407.

[5] C. Chicone, M. Jacobs, Bifurcation of critical periods for plane vector fields, Trans. Amer. Math. Soc. 312 (1989) 433-486.

[6] A. Cima, F. Mañosas, J. Villadelprat, Isochronicity for several classes of hamiltonian systems, J. Differential Equations 157 (1999) 373-413.

[7] J. Françoise, C. Pugh, Keeping track of limit cycles, J. Differential Equations 65 (1986) 139-157.

[8] A. Granata, The problem of differentiating an asymptotic expansion in real powers. Part I: unsatisfactory or partial results by classical approaches, Anal. Math. 36 (2) (2010) 85-112.

[9] A. Granata, The problem of differentiating an asymptotic expansion in real powers. Part II: factorizational theory, Anal. Math. 36 (3) (2010) 173-218.

[10] L. Howland, Note on the Derivative of the Quotient of Two Wronskians, Amer. Math. Monthly 18 (12) (1911) 219-221.

[11] S. Karlin, W. Studden, Tchebycheff systems: With applications in analysis and statistics, Pure and Applied Mathematics, Vol. XV, Interscience Publishers John Wiley \& Sons, New York-London-Sydney, 1966.

[12] W. Loud, Behaviour of the period of solutions of certain plane autonomous systems near centers, Contrib. Differential Equations 3 (1964) 21-36.

[13] F. Mañosas, D. Rojas, J. Villadelprat, The criticality of centers of potential systems at the outer boundary, J. Differential Equations 260 (6) (2016) 4918-4972. 
[14] F. Mañosas, D. Rojas, J. Villadelprat, Study of the period function of a biparametric family of potential centers, Preprint.

[15] F. Mañosas, J. Villadelprat, Bounding the number of zeros of certain Abelian integrals, J. Differential Equations 251 (6) (2011) 1656-1669.

[16] P. Mardešić, D. Marín, M. Saavedra, J. Villadelprat, Unfoldings of saddle-nodes and their dulac time, Preprint.

[17] P. Mardešić, D. Marín, J. Villadelprat, On the time function of the dulac map for families of meromorphic vector fields, Nonlinearity 16 (2003) 855-881.

[18] P. Mardešić, D. Marín, J. Villadelprat, The period function of reversible quadratic centers, J. Differential Equations 224 (2006) 120-171.

[19] P. Mardešić, D. Marín, J. Villadelprat, Unfolding of resonant saddles and the dulac time, Discrete Contin. Dyn. Syst. 21 (2007) 1221-1244.

[20] P. Mardešić, L. Moser-Jauslin, C. Rousseau, Darboux linearization and isochronous centers with a rational first integral, J. Differential Equations 134 (1997) 216-268.

[21] F. Mañosas, J. Villadelprat, A note on the critical periods of potential systems, Internat. J. Bifur. Chaos Appl. Sci. Engrg. 16 (2006) 765-774.

[22] Y. Miyamoto, K. Yagasaki, Monotonicity of the first eigenvalue and the global bifurcation diagram for the branch of interior peak solutions, J. Differential Equations 254 (2013) 342-367.

[23] G. Pólya, On the mean-value theorem corresponding to a given linear homogeneous differential equation, Trans. Amer. Math. Soc. 24 (4) (1922) 312-324.

[24] R. Roussarie, Bifurcation of planar vector fields and hilbert's sixteenth problem, Progr. in Math. 164.

[25] C. Rousseau, B. Toni, Local bifurcations of critical periods in the reduced kukles system, Can. J. Math. 49 (1997) 338-358.

[26] R. Schaaf, A class of hamiltonian systems with increasing periods, J. Reine Angew. Math. 363 (1985) 96-109.

[27] J. Smoller, A. Wasserman, Global bifurcation of steady-state solutions, J. Differential Equations 39 (1981) 269-290.

[28] J. Villadelprat, On the reversible quadratic centers with monotonic period function, Proc. Amer. Math. Soc. 135 (2007) 2555-2565.

[29] K. Yagasaki, Monotonicity of the period function for $u^{\prime \prime}-u+u^{p}=0$ with $p \in \mathbb{R}$ and $p>1$, J. Differential Equations 255 (2013) 1988-2001.

[30] Y.-Q. Ye, C. Y. Lo, Theory of limit cycles, vol. 66, 1984. 